\title{
Nonlinear Model Reduction for Flexible Aircraft Control Design
}

\author{
A. Da Ronch, ${ }^{*}$ K. J. Badcock ${ }^{\dagger}$ \\ University of Liverpool, Liverpool, England L69 3BX, United Kingdom \\ Y. Wang, $\ddagger$ A. Wynn, $§$ and R. Palacios ฯ \\ Imperial College, London, England SW7 2AZ, United Kingdom
}

\begin{abstract}
The paper describes a systematic approach to the model reduction of large dimension fluid-structure-flight models, and the subsequent flight control design of very flexible aircraft. System nonlinearities may be due to the large wing deformations, the coupling between flexible and rigid body dynamics and/or flow separation at large angles of incidence. A nonlinear reduced order model is used to reduce the computational cost and dimension of the large-order nonlinear system for a practical control law design. The approach uses information on the eigenspectrum of the coupled system Jacobian matrix and projects the system through a series expansion onto a small basis of eigenvectors representative of the full-order dynamics. For a pitch-plunge aerofoil with structural nonlinearities, a controller based on reduced models was designed to alleviate gust loads. The approach to model reduction was also demonstrated for a two-dimensional problem with aerodynamics modelled using the computational fluid dynamics equations, and a flexible wing modelled using the geometrically-exact nonlinear beam equations. In all cases, the model reduction was found adequate to predict the large order system dynamics at a neglegible cost compared to that incurred by solving the nonlinear full-order system.
\end{abstract}

\section{Nomenclature}

\begin{tabular}{|c|c|}
\hline$A$ & $=$ Jacobian matrix of $\boldsymbol{R}$ with respect to $\boldsymbol{w}$ \\
\hline$B, C$ & $=$ second and third Jacobian operators \\
\hline$b$ & $=$ semichord \\
\hline$C_{\xi}, C_{\alpha}$ & $=$ viscous damping in plunge and pitch, respectively \\
\hline$C_{\xi}^{c}$ & $=$ critical damping in plunge, $2 \sqrt{m K_{\xi}}$ \\
\hline$C_{\alpha}^{c}$ & $=$ critical damping in pitch, $2 \sqrt{I_{\alpha} K_{\alpha}}$ \\
\hline$K_{\xi}, K_{\alpha}$ & $=$ plunge stiffness and torsional stiffness about elastic axis \\
\hline$I_{\alpha}$ & $=$ second moment of inertia of aerofoil about elastic axis \\
\hline$C_{L}, C_{m}$ & $=$ lift and pitch moment coefficients \\
\hline$h_{g}$ & $=$ gust gradient \\
\hline$L, M$ & $=$ lift and pitch moment \\
\hline$m$ & $=$ aerofoil sectional mass \\
\hline$S_{\alpha}$ & $=$ first moment of inertia of aerofoil about elastic axis \\
\hline$t$ & $=$ physical time \\
\hline$x_{\alpha}$ & $=$ aerofoil static unbalance, $S_{\alpha} / m b$ \\
\hline $\boldsymbol{R}$ & $=$ residual vector \\
\hline
\end{tabular}

${ }^{*}$ Research Associate, School of Engineering. Member AIAA (Corresponding Author: A.Da-Ronch@liverpool.ac.uk).

${ }^{\dagger}$ Professor, School of Engineering. Senior Member AIAA.

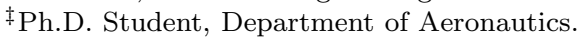

$\S$ Research Associate, Department of Aeronautics.

ISenior Lecturer, Department of Aeronautics. Member AIAA. 


$r_{a}$
$U$
$U_{L}$
$U^{*}$
$\boldsymbol{w}$
$W_{g}$
$W_{0}$

Greek
$\alpha$
$\beta_{\xi}, \beta_{\xi_{5}}, \beta_{\alpha}, \beta_{\alpha_{5}}$
$\lambda_{i}$
$\tau$
$\omega_{\xi}$
$\omega_{\alpha}$
$\phi_{i}, \boldsymbol{\psi}_{i}$
$\bar{\omega}$
$\zeta_{\xi}$
$\zeta_{\alpha}$
$\xi$
$\mu$
$\rho$

Symbol

()

$$
\begin{aligned}
& =\text { radius of gyration of aerofoil about elastic axis, } r_{a}^{2}=I_{\alpha} / m b^{2} \\
& =\text { freestream velocity } \\
& =\text { linear flutter speed } \\
& =\text { reduced velocity, } U / b \omega_{\alpha} \\
& =\text { vector of unknowns } \\
& =\text { gust vertical velocity } \\
& =\text { intensity of gust vertical velocity }
\end{aligned}
$$

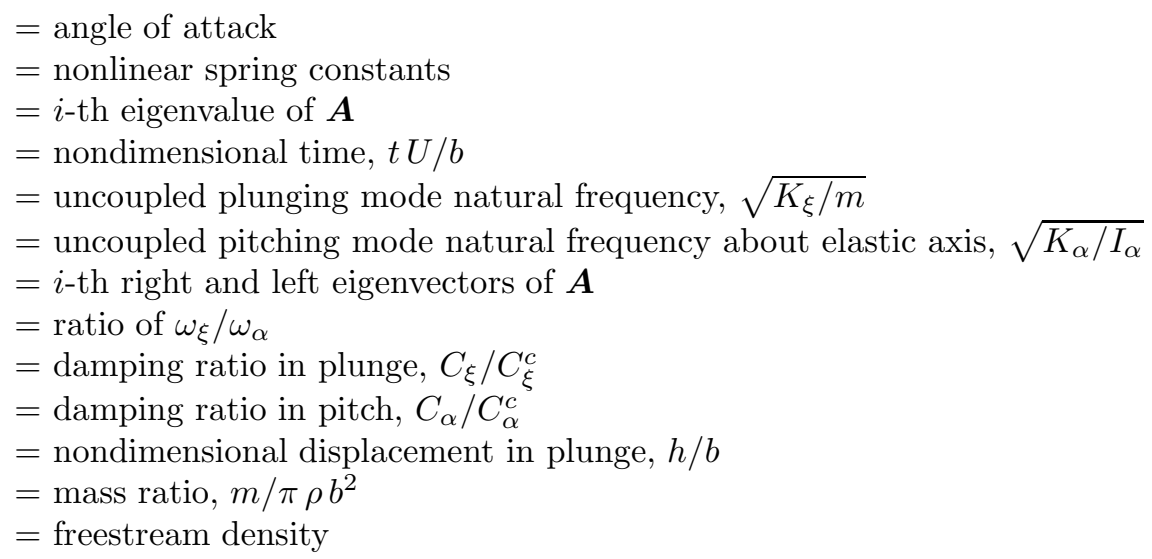

\section{Introduction}

The work detailed in this paper is part of the development of a systematic approach to flight control system (FCS) design for very flexible or very large aircraft. Examples of vehicles in this class are those considered for low-environmental impact air transport and for long-endurance unmanned operations. Improved aircraft performance is generally achieved through lightweight solutions with high aspect ratio wings for maximum aerodynamic efficiency. The combination of low structural weight fraction and high aerodynamic efficiency yields inherently flexible wings with a nonlinear structural and flight dynamics behaviour. The traditional separation of aeroelasticity and flight dynamics is therefore not appropriate for flight control when low structural frequencies, which are often associated with large amplitude motions, are present. The mishap of NASA's Helios aircraft demonstrated that (linear) traditional design methods are no longer adequate for the analysis of the next-generation aircraft. ${ }^{1}$ Modelling and design methods based on a fully coupled system analysis are therefore necessary. ${ }^{2}$

A consideration is that model reduction methods assume linearity or, at most, weak nonlinearities. ${ }^{3}$ The development of nonlinear ROMs is an area that urgently needs advances, in general, and is necessary for control applications of flexible aircraft, in particular. There are two approaches to model reduction. System identification methods take the response of the system to known inputs, and use this information to build a low-order model. ${ }^{4,5}$ The disadvantages of these methods are the lack of a general robust parametrization of the model and the inability to predict any physics that is not included in the training data. However, these methods have been applied successfully. ${ }^{6}$ The second approach is to manipulate the full-order nonlinear residual to reduce the cost of calculations. The advantage in doing this is that the predictive capability of the full-order model (FOM) is retained. The disadvantage is the added technical complication of manipulating the system. An example is the harmonic balance method, which has been exploited for dynamic derivative predictions avoiding costly time-accurate CFD runs. ${ }^{7}$ This second approach is considered in the current paper. 
For limit-cycle oscillation (LCO) prediction, the system responds in the critical mode close to the bifurcation point. One approach presented in Refs. ${ }^{8,9}$ is to project the full-order model onto the critical mode and expand the residual in Taylor series, retaining quadratic and cubic terms. The influence of the noncritical space on the critical mode is included through a center manifold approximation. The method has been successfully applied to various testcases, including the LCO prediction dominated by the motion of a shock wave $^{8}$ and a prototype flight dynamics instability of a delta wing. ${ }^{9}$

Control of a two-dimensional aeroelastic system with structural nonlinearities has been reported by several researchers. For example, Ref. ${ }^{10}$ used a nonlinear feedback control law based on the state-dependent Riccati equation, and Ref. ${ }^{11}$ applied a partial feedback linearization to control LCO of a wind tunnel model. However, standard linear control design methods may be inadequate for highly flexible aircraft because the dynamic behaviour is intrinsically nonlinear. New approaches to nonlinear FCS design are required to control these systems in a provably robust manner. The contribution of this paper is to develop an approach to model reduction that is capable of producing a nonlinear reduced order model for the dynamics of a flexible aircraft.

The paper continues with an overview of the nonlinear coupled system of equations. The model reduction and the control formulation are then considered, followed by a description of the algorithm used in calculating the Jacobian operators. A pitch-plunge aerofoil with structural nonlinearity, an aerofoil with aerodynamics governed by the Euler equations, and a flexible wing modelled using the geometrically-exact nonlinear beam equations are the testcases.

\section{Large-Order Nonlinear Model}

The fully coupled nonlinear model for the description of the flight dynamics of a very flexible aircraft can be represented in a state-space form. Denote by $\boldsymbol{w}$ the $n$-dimensional state-space vector which is conveniently partitioned into fluid, structural and rigid body degrees of freedom

$$
\boldsymbol{w}=\left[\boldsymbol{w}_{f}^{T}, \boldsymbol{w}_{s}^{T}, \boldsymbol{w}_{r}^{T}\right]^{T}
$$

In the case that CFD is used as the source of the aerodynamic predictions, the vector $\boldsymbol{w}_{f}$ may contain millions of unknowns. The state-space equations in the general vector form are

$$
\frac{d \boldsymbol{w}}{d t}=\boldsymbol{R}\left(\boldsymbol{w}, \boldsymbol{u}_{c}, \boldsymbol{u}_{d}\right)
$$

where $\boldsymbol{R}$ is the (nonlinear) residual, $\boldsymbol{u}_{c}$ is the input vector, and $\boldsymbol{u}_{d}$ is the exogenous vector for the description of some form of disturbance acting on the system. The homogeneous system has an equilibrium point, $\boldsymbol{w}_{0}$, for given constant $\boldsymbol{u}_{c 0}$ and $\boldsymbol{u}_{d 0}$ corresponding to a constant solution in the state space and satisfying

$$
\frac{d \boldsymbol{w}_{0}}{d t}=\boldsymbol{R}\left(\boldsymbol{w}_{0}, \boldsymbol{u}_{c 0}, \boldsymbol{u}_{d 0}\right)=\mathbf{0}
$$

The residual form in Eq. (2) forms the basis for the model reduction described below. The system is often parametrized in terms of an independent parameter (freestream speed, air density, altitude, etc.) for stability analysis.

\section{Nonlinear Model Reduction}

Denote $\Delta \boldsymbol{w}=\boldsymbol{w}-\boldsymbol{w}_{0}$ the increment in the state-space vector with respect to an equilibrium solution. The large-order nonlinear residual formulated in Eq. (2) is expanded in a Taylor series around the equilibrium point

$$
\boldsymbol{R}(\boldsymbol{w}) \approx \boldsymbol{A} \Delta \boldsymbol{w}+\frac{\partial \boldsymbol{R}}{\partial \boldsymbol{u}_{c}} \Delta \boldsymbol{u}_{c}+\frac{\partial \boldsymbol{R}}{\partial \boldsymbol{u}_{d}} \Delta \boldsymbol{u}_{d}+\frac{1}{2} \boldsymbol{B}(\Delta \boldsymbol{w}, \Delta \boldsymbol{w})+\frac{1}{6} \boldsymbol{C}(\Delta \boldsymbol{w}, \Delta \boldsymbol{w}, \Delta \boldsymbol{w})+\mathcal{O}\left(|\Delta \boldsymbol{w}|^{4}\right)
$$

retaining terms up to third order in the perturbation variable. The Jacobian matrix of the system is denoted as $\boldsymbol{A}$ and the vectors $\boldsymbol{B}$ and $\boldsymbol{C}$ indicate, respectively, the second and third order Jacobian operators. The 
elements are calculated as

$$
\begin{aligned}
A_{i j} & =\frac{\partial R_{i}\left(\boldsymbol{w}_{0}\right)}{\partial w_{j}} \\
B_{i}(\boldsymbol{x}, \boldsymbol{y}) & =\sum_{j, k} \frac{\partial^{2} R_{i}\left(\boldsymbol{w}_{0}\right)}{\partial w_{j} \partial w_{k}} x_{j} y_{k} \\
C_{i}(\boldsymbol{x}, \boldsymbol{y}, \boldsymbol{z}) & =\sum_{j, k, l} \frac{\partial^{3} R_{i}\left(\boldsymbol{w}_{0}\right)}{\partial w_{j} \partial w_{k} \partial w_{l}} x_{j} y_{k} z_{l}
\end{aligned}
$$

The full-order system is projected onto a basis formed by a small number (denoted by $m$ ) of eigenvectors of the Jacobian matrix evaluated at the equilibrium position. A rational choice would be to retain only the slow modes since they are likely to dominate the system dynamics, with the exception of unstable fast modes leading to flutter. The right and left eigenvalues and eigenvectors are complex in general. The eigenvalues of $\boldsymbol{A}$ are the same as the eigenvalues of $\boldsymbol{A}^{T}$, whereas the eigenvectors of $\boldsymbol{A}$ are different from the eigenvectors of $\boldsymbol{A}^{T}$. The set of right eigenvectors $\phi_{i}$ are obtained by solving

$$
\boldsymbol{A} \boldsymbol{\phi}_{i}=\lambda_{i} \boldsymbol{\phi}_{i} \text { for } i=1, \ldots, n
$$

The set of left eigenvectors, $\boldsymbol{\psi}_{i}$, are obtained by solving the adjoint eigenvalue problem

$$
\boldsymbol{A}^{T} \boldsymbol{\psi}_{i}=\bar{\lambda}_{i} \boldsymbol{\psi}_{i} \text { for } i=1, \ldots, n
$$

Details on how the eigenvalue problem is solved for large-order aeroelastic systems are given in $\S$ VI. If all the eigenvalues are distinct, the right and left eigenvectors corresponding to different eigenvalues are biorthogonal. It is then convenient to normalize the eigenvectors so as to satisfy the biorthonormality conditions, expressed by

$$
<\phi_{i}, \phi_{i}>=1, \quad<\boldsymbol{\psi}_{j}, \phi_{i}>=\delta_{i j}, \quad<\boldsymbol{\psi}_{j}, \overline{\boldsymbol{\phi}}_{i}>=0 \quad \text { for } i, j=1, \ldots, m
$$

and resulting in

$$
<\boldsymbol{\psi}_{j}, \boldsymbol{A} \boldsymbol{\phi}_{i}>=\lambda_{i} \delta_{i j}, \quad<\boldsymbol{\psi}_{j}, \boldsymbol{A} \overline{\boldsymbol{\phi}}_{i}>=0 \text { for } i, j=1, \ldots, m
$$

where $\delta_{i j}$ is the Kronecker delta. Note that the Hermitian inner product is defined as $\langle\boldsymbol{x}, \boldsymbol{y}\rangle=\overline{\boldsymbol{x}}^{T} \boldsymbol{y}$, with the overbar denoting complex conjugation. The $(n \times m)$ right and left modal matrices, respectively, $\boldsymbol{\Phi}$ and $\boldsymbol{\Psi}$, are formed as

$$
\boldsymbol{\Phi}=\left[\phi_{1}, \ldots, \phi_{m}\right], \quad \boldsymbol{\Psi}=\left[\boldsymbol{\psi}_{1}, \ldots, \boldsymbol{\psi}_{m}\right]
$$

The full order model is projected onto a small basis of $m$ representative eigenvectors using a transformation of coordinates

$$
\Delta w=\Phi z+\bar{\Phi} \bar{z}
$$

where $\boldsymbol{z} \in \mathbb{C}^{m}$ is the state-space vector governing the dynamics of the reduced-order nonlinear system.

When nonlinear terms in the Taylor series expansion of the large-order nonlinear residual are neglected, a linear reduced model can be derived. Substituting the transformation of coordinates in Eq. (11) into Eq. (4) and premultiplying each term by the conjugate transpose of the left modal matrix yields

$$
\overline{\boldsymbol{\psi}}_{j}^{T}\left(\boldsymbol{\phi}_{i} z_{i}^{\prime}+\overline{\boldsymbol{\phi}}_{i} \bar{z}_{i}^{\prime}\right)=\overline{\boldsymbol{\psi}}_{j}^{T}\left(\boldsymbol{A} \boldsymbol{\phi}_{i} z_{i}+\boldsymbol{A} \overline{\boldsymbol{\phi}}_{i} \bar{z}_{i}+\frac{\partial \boldsymbol{R}}{\partial \boldsymbol{u}_{c}} \Delta \boldsymbol{u}_{c}+\frac{\partial \boldsymbol{R}}{\partial \boldsymbol{u}_{d}} \Delta \boldsymbol{u}_{d}\right) \quad \text { for } i, j=1, \ldots, m
$$

If the eigenvalues are distinct, which is not always the case, the properties in Eqs. (8) and (9) yield the formulation of a linear $\mathrm{ROM}$

$$
z_{i}^{\prime}=\lambda_{i} z_{i}+\overline{\boldsymbol{\psi}}_{i}^{T}\left(\frac{\partial \boldsymbol{R}}{\partial \boldsymbol{u}_{c}} \Delta \boldsymbol{u}_{c}+\frac{\partial \boldsymbol{R}}{\partial \boldsymbol{u}_{d}} \Delta \boldsymbol{u}_{d}\right) \quad \text { for } i=1, \ldots, m
$$

The set of equations in Eq. (13) consists of $m$ uncoupled ordinary differential equations (ODEs). The terms of the reduced model are calculated once and for all after the eigenvalues, eigenvectors, and equilibrium are known. 
Manipulation of the higher-order terms in Eq. (4) yields the formulation of a nonlinear ROM. In addition to the linear terms in Eq. (12), the two contributions from the second and third Jacobian operators are

$$
\overline{\boldsymbol{\psi}}_{j}^{T}\left(\frac{1}{2} B_{i}(\Delta \boldsymbol{w}, \Delta \boldsymbol{w})+\frac{1}{6} C_{i}(\Delta \boldsymbol{w}, \Delta \boldsymbol{w}, \Delta \boldsymbol{w})\right)
$$

The terms $\boldsymbol{B}$ and $\boldsymbol{C}$ are, respectively, bilinear and trilinear functions in the argument variables. This property implies that, after substitution of the transformation of coordinates, the additional terms may be written as

$$
\begin{aligned}
& B_{i}(\Delta \boldsymbol{w}, \Delta \boldsymbol{w})=\sum_{r=1}^{m} \sum_{s=1}^{m}\left(B_{i}\left(\phi_{r}, \phi_{s}\right) z_{r} z_{s}+B_{i}\left(\phi_{r}, \bar{\phi}_{s}\right) z_{r} \bar{z}_{s}+\right. \\
& \left.B_{i}\left(\bar{\phi}_{r}, \phi_{s}\right) \bar{z}_{r} z_{s}+B_{i}\left(\bar{\phi}_{r}, \bar{\phi}_{s}\right) \bar{z}_{r} \bar{z}_{s}\right)
\end{aligned}
$$

and

$$
\begin{array}{r}
C_{i}(\Delta \boldsymbol{w}, \Delta \boldsymbol{w}, \Delta \boldsymbol{w})=\sum_{r=1}^{m} \sum_{s=1}^{m} \sum_{t=1}^{m}\left(C_{i}\left(\boldsymbol{\phi}_{r}, \phi_{s}, \phi_{t}\right) z_{r} z_{s} z_{t}+C_{i}\left(\phi_{r}, \phi_{s}, \bar{\phi}_{t}\right) z_{r} z_{s} \bar{z}_{t}+\right. \\
C_{i}\left(\phi_{r}, \bar{\phi}_{s}, \phi_{t}\right) z_{r} \bar{z}_{s} z_{t}+C_{i}\left(\phi_{r}, \bar{\phi}_{s}, \bar{\phi}_{t}\right) z_{r} \bar{z}_{s} \bar{z}_{t}+ \\
C_{i}\left(\bar{\phi}_{r}, \phi_{s}, \phi_{t}\right) \bar{z}_{r} z_{s} z_{t}+C_{i}\left(\bar{\phi}_{r}, \phi_{s}, \bar{\phi}_{t}\right) \bar{z}_{r} z_{s} \bar{z}_{t}+ \\
\left.C_{i}\left(\bar{\phi}_{r}, \bar{\phi}_{s}, \phi_{t}\right) \bar{z}_{r} \bar{z}_{s} z_{t}+C_{i}\left(\bar{\phi}_{r}, \bar{\phi}_{s}, \bar{\phi}_{t}\right) \bar{z}_{r} \bar{z}_{s} \bar{z}_{t}\right)
\end{array}
$$

The second and third order Jacobians consist, in general, of $4 \mathrm{~m}^{2}$ and $8 \mathrm{~m}^{3}$ contributions. However, it is possible to exploit the symmetry of the Jacobians with respect to the arguments ${ }^{\text {a }}$, which reduces the total number of evaluations to $2 m^{2}+m$ in the case of the bilinear function. Equation (15) can then be rearranged as

$$
\begin{array}{r}
B_{i}(\Delta \boldsymbol{w}, \Delta \boldsymbol{w})=\sum_{r=1}^{m}\left(B_{i}\left(\phi_{r}, \phi_{r}\right) z_{r}^{2}+2 B_{i}\left(\phi_{r}, \bar{\phi}_{r}\right) z_{r} \bar{z}_{r}+B_{i}\left(\overline{\boldsymbol{\phi}}_{r}, \overline{\boldsymbol{\phi}}_{r}\right) \bar{z}_{r}^{2}+\right. \\
2 \sum_{s=r+1}^{m}\left(B_{i}\left(\phi_{r}, \phi_{s}\right) z_{r} z_{s}+B_{i}\left(\phi_{r}, \bar{\phi}_{s}\right) z_{r} \bar{z}_{s}+\right. \\
\left.\left.B_{i}\left(\bar{\phi}_{r}, \phi_{s}\right) \bar{z}_{r} z_{s}+B_{i}\left(\bar{\phi}_{r}, \bar{\phi}_{s}\right) \bar{z}_{r} \bar{z}_{s}\right)\right)
\end{array}
$$

For the third order Jacobian term, the total number of evaluations may be reduced to $2 / 3\left(2 m^{3}+3 m^{2}+m\right)$. For conciseness, the corresponding formulation of $\boldsymbol{C}$ is omitted.

The high-order Jacobian terms required in the model reduction are represented by the bilinear and trilinear functionals formulated in Eq. (5). It is possible to calculate all the contributions without having to resort to complex arithmetic, or to calculating all the second and third order partial derivatives analytically. ${ }^{8}$ Because it is only their action on vectors that is required, matrix-free products are used.

For the first order Jacobian-vector product and for the second and third Jacobian operators, the directional derivatives on any set of coinciding real vectors, $\boldsymbol{x} \in \mathbb{R}^{n}$, can be approximated using finite differences

$$
\begin{aligned}
\boldsymbol{A} \boldsymbol{x} & =\frac{\boldsymbol{R}_{1}-\boldsymbol{R}_{-1}}{2 \epsilon}+\mathcal{O}\left(\epsilon^{2}\right) \\
\boldsymbol{B}(\boldsymbol{x}, \boldsymbol{x}) & =\frac{\boldsymbol{R}_{1}-2 \boldsymbol{R}_{0}+\boldsymbol{R}_{-1}}{\epsilon^{2}}+\mathcal{O}\left(\epsilon^{3}\right) \\
\boldsymbol{C}(\boldsymbol{x}, \boldsymbol{x}, \boldsymbol{x}) & =\frac{-\boldsymbol{R}_{3}+8 \boldsymbol{R}_{2}-13 \boldsymbol{R}_{1}+13 \boldsymbol{R}_{-1}-8 \boldsymbol{R}_{-2}+\boldsymbol{R}_{-3}}{8 \epsilon^{3}}+\mathcal{O}\left(\epsilon^{4}\right)
\end{aligned}
$$

where $\boldsymbol{R}_{l}=\boldsymbol{R}\left(\boldsymbol{x}_{0}+l \epsilon \Delta \boldsymbol{x}\right)$. Note that the system Jacobian matrix is in general available in analytic form. To calculate all the terms in Eqs. (15) and (16), a set of identities for the manipulation of terms like $\boldsymbol{B}(\boldsymbol{x}, \boldsymbol{y})$

${ }^{\text {a Note that }} B_{i}(\boldsymbol{x}, \boldsymbol{y})=B_{i}(\boldsymbol{y}, \boldsymbol{x})$ and similar properties hold for the third order Jacobian. 
and $\boldsymbol{C}(\boldsymbol{x}, \boldsymbol{y}, \boldsymbol{z})$ can be derived. The following two identities

$$
\begin{aligned}
& \boldsymbol{B}(\boldsymbol{x}+\boldsymbol{y}, \boldsymbol{x}+\boldsymbol{y})=\boldsymbol{B}(\boldsymbol{x}, \boldsymbol{x})+2 \boldsymbol{B}(\boldsymbol{x}, \boldsymbol{y})+\boldsymbol{B}(\boldsymbol{y}, \boldsymbol{y}) \\
& \boldsymbol{B}(\boldsymbol{x}-\boldsymbol{y}, \boldsymbol{x}-\boldsymbol{y})=\boldsymbol{B}(\boldsymbol{x}, \boldsymbol{x})-2 \boldsymbol{B}(\boldsymbol{x}, \boldsymbol{y})+\boldsymbol{B}(\boldsymbol{y}, \boldsymbol{y})
\end{aligned}
$$

yield the desired result for the second Jacobian term

$$
\boldsymbol{B}(\boldsymbol{x}, \boldsymbol{y})=\frac{1}{4}(\boldsymbol{B}(\boldsymbol{x}+\boldsymbol{y}, \boldsymbol{x}+\boldsymbol{y})-\boldsymbol{B}(\boldsymbol{x}-\boldsymbol{y}, \boldsymbol{x}-\boldsymbol{y}))
$$

A similar set of identities is readily derived for $\boldsymbol{C}$ which combined together results in the following general formulation for a third order Jacobian term

$$
\begin{gathered}
\boldsymbol{C}(\boldsymbol{x}, \boldsymbol{y}, \boldsymbol{z})=\frac{1}{6}(\boldsymbol{C}(\boldsymbol{x}+\boldsymbol{y}+\boldsymbol{z}, \boldsymbol{x}+\boldsymbol{y}+\boldsymbol{z}, \boldsymbol{x}+\boldsymbol{y}+\boldsymbol{z})-\boldsymbol{C}(\boldsymbol{x}+\boldsymbol{y}, \boldsymbol{x}+\boldsymbol{y}, \boldsymbol{x}+\boldsymbol{y})- \\
\boldsymbol{C}(\boldsymbol{x}+\boldsymbol{z}, \boldsymbol{x}+\boldsymbol{z}, \boldsymbol{x}+\boldsymbol{z})-\boldsymbol{C}(\boldsymbol{y}+\boldsymbol{z}, \boldsymbol{y}+\boldsymbol{z}, \boldsymbol{y}+\boldsymbol{z})+ \\
\boldsymbol{C}(\boldsymbol{x}, \boldsymbol{x}, \boldsymbol{x})+\boldsymbol{C}(\boldsymbol{y}, \boldsymbol{y}, \boldsymbol{y})+\boldsymbol{C}(\boldsymbol{z}, \boldsymbol{z}, \boldsymbol{z}))
\end{gathered}
$$

Because eigenvalues are complex in general, the formulations in Eqs. (23) and (24) derived for any real vector, $\boldsymbol{x}, \boldsymbol{y}, \boldsymbol{z} \in \mathbb{R}^{n}$, can be applied to any complex vector when the real and imaginary parts are treated separately. Denoting

$$
\boldsymbol{p}=\boldsymbol{p}_{1}+i \boldsymbol{p}_{2}, \quad \boldsymbol{p} \in \mathbb{C}^{n}, \quad \boldsymbol{p}_{1}, \boldsymbol{p}_{2} \in \mathbb{R}^{n}
$$

it follows that, for example,

$$
\boldsymbol{B}(\boldsymbol{p}, \boldsymbol{p})=\boldsymbol{B}\left(\boldsymbol{p}_{1}, \boldsymbol{p}_{1}\right)-\boldsymbol{B}\left(\boldsymbol{p}_{2}, \boldsymbol{p}_{2}\right)+2 i \boldsymbol{B}\left(\boldsymbol{p}_{1}, \boldsymbol{p}_{2}\right)
$$

and

$$
\boldsymbol{C}(\boldsymbol{p}, \boldsymbol{p}, \boldsymbol{p})=\boldsymbol{C}\left(\boldsymbol{p}_{1}, \boldsymbol{p}_{1}, \boldsymbol{p}_{1}\right)-3 \boldsymbol{C}\left(\boldsymbol{p}_{1}, \boldsymbol{p}_{2}, \boldsymbol{p}_{2}\right)+i\left(3 \boldsymbol{C}\left(\boldsymbol{p}_{1}, \boldsymbol{p}_{1}, \boldsymbol{p}_{2}\right)-\boldsymbol{C}\left(\boldsymbol{p}_{2}, \boldsymbol{p}_{2}, \boldsymbol{p}_{2}\right)\right)
$$

The evaluation of the finite differences suffers from the truncation error for values of the step size $\epsilon$ which are too large, and from the rounding error for values which are too small. The latter effect is more significant for the coefficients that include a third Jacobian product. References ${ }^{8,9}$ conducted convergence studies and obtained a reliable set of coefficients for the reduced model over a significant range of $\epsilon$.

\section{Robust Control Design}

To formulate a control problem, Eq. (13) is written in matrix form

$$
\boldsymbol{z}^{\prime}=\boldsymbol{\Lambda} \boldsymbol{z}+\boldsymbol{M}_{B} \boldsymbol{u}_{c}+\boldsymbol{M}_{E} \boldsymbol{u}_{d}
$$

Here, $\boldsymbol{z}$ is a vector containing the states $z_{i}, \boldsymbol{\Lambda}$ is the diagonal matrix containing the eigenvalues $\lambda_{i}, \boldsymbol{M}_{B}$ contains coefficients for the control input, and $\boldsymbol{M}_{E}$ is the coefficient matrix for the gust disturbance.

For two-degree-of-freedom aerofoil control problems with a single control input, as in Section V, $\boldsymbol{u}_{c}$ consists of the value of the input and its two derivatives with time

$$
\boldsymbol{u}_{c}=\left[\delta, \delta^{\prime}, \delta^{\prime \prime}\right]^{T}
$$

Since the three functions are mutually dependent, it is convenient to incorporate $\delta$ and $\delta^{\prime}$ into an extended state vector

$$
\boldsymbol{x}=\left[\boldsymbol{z}^{T}, \delta, \delta^{\prime}\right]^{T}
$$

Defining $\boldsymbol{u}=\delta^{\prime \prime}$ to be the control input, Eq. (28) now has the form

$$
\begin{aligned}
\boldsymbol{x}^{\prime}=\left(\begin{array}{c}
\boldsymbol{z} \\
\delta \\
\delta^{\prime}
\end{array}\right)^{\prime} & =\left[\begin{array}{ccc}
\boldsymbol{\Lambda} & \boldsymbol{M}_{B 0} & \boldsymbol{M}_{B 1} \\
\mathbf{0} & 0 & 1 \\
\mathbf{0} & 0 & 0
\end{array}\right]\left(\begin{array}{c}
\boldsymbol{z} \\
\delta \\
\delta^{\prime}
\end{array}\right)+\left[\begin{array}{c}
\boldsymbol{M}_{B 2} \\
0 \\
1
\end{array}\right] \delta^{\prime \prime}+\left[\begin{array}{c}
\boldsymbol{M}_{E} \\
0 \\
0
\end{array}\right] \boldsymbol{u}_{d} \\
& =\hat{\boldsymbol{M}}_{A} \boldsymbol{x}+\hat{\boldsymbol{M}}_{B} \boldsymbol{u}+\hat{\boldsymbol{M}}_{E} \boldsymbol{u}_{d}
\end{aligned}
$$


The controlled and measured outputs are taken to be linear combinations of elements of $\Delta \boldsymbol{w}$, that gives,

$$
\boldsymbol{y}_{\text {meas }}=\boldsymbol{M}_{C} \boldsymbol{x}
$$

As the output is linear only with the real and imaginary parts of the states separately, a linear controller formulation requires splitting the complex states into their real and imaginary parts

$$
\tilde{\boldsymbol{x}}=\left(\begin{array}{c}
\operatorname{Re}(\boldsymbol{x}) \\
\operatorname{Im}(\boldsymbol{x})
\end{array}\right), \quad \boldsymbol{y}_{c t r l}=\left(\begin{array}{c}
\alpha \\
y_{u}
\end{array}\right), \quad \tilde{\boldsymbol{w}}=\left(\begin{array}{c}
\boldsymbol{u}_{d} \\
w_{d}
\end{array}\right)
$$

where the outputs are distinguished by what the controller is aiming to control, $\boldsymbol{y}_{\text {ctrl }}$, and what the controller actually has information about, $\boldsymbol{y}_{\text {meas }}$. Constants $y_{u}$ and $w_{d}$ are artificial output and disturbance included to provide tuning variables for the $\boldsymbol{H}^{\infty}$ controller. The state-space system can now be written in the standard form for $\boldsymbol{H}^{\infty}$ control

$$
\left(\begin{array}{c}
\tilde{\boldsymbol{x}}^{\prime} \\
\boldsymbol{y}_{\text {ctrl }} \\
\boldsymbol{y}_{\text {meas }}
\end{array}\right)=\left[\begin{array}{ccc}
\tilde{\boldsymbol{M}}_{A} & \tilde{\boldsymbol{M}}_{E} & \tilde{\boldsymbol{M}}_{B} \\
\boldsymbol{C}_{1} & \boldsymbol{D}_{11} & \boldsymbol{D}_{12} \\
\boldsymbol{C}_{2} & \boldsymbol{D}_{21} & \boldsymbol{D}_{22}
\end{array}\right]\left(\begin{array}{c}
\tilde{\boldsymbol{x}} \\
\tilde{\boldsymbol{w}} \\
\boldsymbol{u}
\end{array}\right)
$$

where the matrices are expanded in the same way

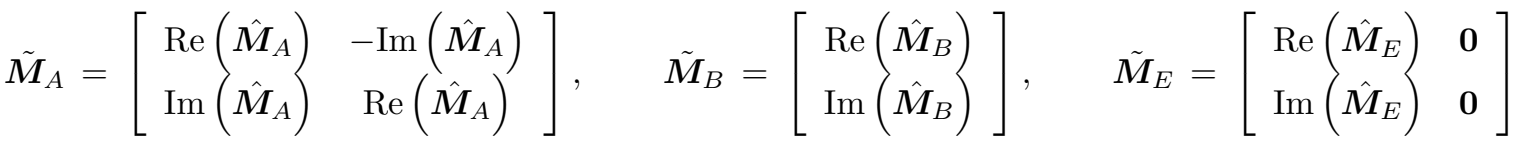

$$
\begin{aligned}
& \boldsymbol{C}_{1}=\left[\begin{array}{c}
\boldsymbol{M}_{C} \\
\mathbf{0}
\end{array}\right], \quad \boldsymbol{C}_{2}=\boldsymbol{M}_{C} \\
& \boldsymbol{D}_{11}=\mathbf{0}, \quad \boldsymbol{D}_{12}=\left[\begin{array}{c}
\mathbf{0} \\
k_{c}
\end{array}\right], \quad \boldsymbol{D}_{21}=\left[\begin{array}{ll}
\mathbf{0} & k_{d}
\end{array}\right], \quad \boldsymbol{D}_{22}=\mathbf{0}
\end{aligned}
$$

Here, $k_{c}$ and $k_{d}$ are the tuning parameters for the $\boldsymbol{H}^{\infty}$ controller. The parameter $k_{c}$ is a weighting function that feeds the controlled output via $y_{u}=k_{c} \boldsymbol{u}$, so that the magnitude of the control input is itself penalised. Similarly $k_{d}$ is a weighting function on a virtual disturbance $w_{d}$ and is used to simulate the degree of uncertainty in the controller measurements $\boldsymbol{y}_{\text {meas }}$.

The resulting controller has the form

$$
\boldsymbol{u}(s)=\boldsymbol{K}(s) \boldsymbol{y}_{\text {meas }}(s)
$$

where $\boldsymbol{K}(s)$ is the transfer function of the $\boldsymbol{H}^{\infty}$ controller in the Laplace domain. This controller is the one that minimises the $\boldsymbol{H}^{\infty}$-norm of the transfer function taking the disturbance signal $\tilde{\boldsymbol{w}}$ to the controlled output $\boldsymbol{y}_{c t r l}$, by creating a controller that uses information from $\boldsymbol{y}_{\text {meas }}$ to change the input $\boldsymbol{u}$. By varying the tuning parameters, the $\boldsymbol{H}^{\infty}$ controller can be synthesised with variable weights on states (how much should the states be stabilised) relative to the input (effectively limiting the range of inputs) and measurement noise (reflecting confidence in the measured output).

Having designed the controller using the linearized system in Eq. (28), the control law in Eq. (36) can be applied to the nonlinear reduced order model via

$$
\begin{aligned}
\boldsymbol{x}^{\prime} & =\hat{\boldsymbol{M}}_{A} \boldsymbol{x}+\left[\begin{array}{lll}
f(\boldsymbol{z})^{T} & 0 & 0
\end{array}\right]^{T}+\hat{\boldsymbol{M}}_{B} \boldsymbol{u}+\hat{\boldsymbol{M}}_{E} \boldsymbol{u}_{d} \\
\boldsymbol{y}_{\text {meas }} & =\boldsymbol{M}_{C} \boldsymbol{x} \\
\boldsymbol{u}(s) & =\boldsymbol{K}(s) \boldsymbol{y}_{\text {meas }}
\end{aligned}
$$

Here the additional $f(\boldsymbol{z})$ term describes the nonlinear part of the open-loop dynamics. The controller dynamics are still linear and remain the same as Eq. (36). It will be shown later that the linear controller still performs well when faced with this particular nonlinear system, for realistic amplitudes of disturbances. 


\section{Pitch-Plunge Aerofoil with Structural Nonlinearity}

The aerofoil shown in Fig. 1 has two degrees of freedom that define the motion about a reference elastic axis (e.a.). The plunge deflection is denoted by $h$, positive downward, and $\alpha$ is the angle of attack about the elastic axis, positive with nose up. The aerofoil is equipped with a massless trailing-edge flap with hinge at a distance $c b$ from the midchord. The flap deflection, $\delta$, is defined relative to the undeflected position and not relative to the wind direction. The motion is restrained by two springs, $K_{\xi}$ and $K_{\alpha}$, and is assumed to have a horizontal equilibrium position at $h=\alpha=\delta=0$. The system also contains structural damping in both degrees of freedom.

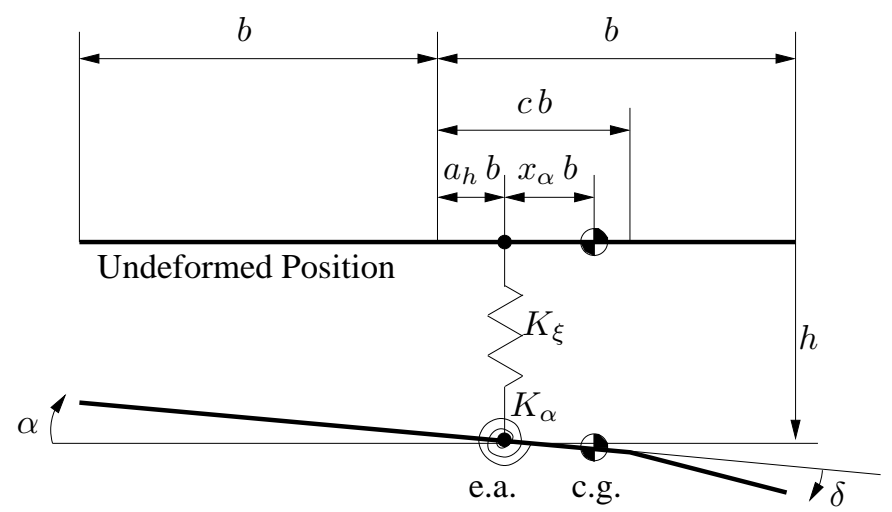

Figure 1. Schematic of an aerofoil section with trailing-edge flap; the wind velocity is to the right and horizontal

The equations of motion in dimensional form with nonlinear restoring forces in pitch and plunge can be derived, for example, using the Lagrange equations ${ }^{12}$

$$
\begin{aligned}
& m \ddot{h}+S_{\alpha} \ddot{\alpha}+C_{\xi} \dot{h}+K_{\xi}\left(h+\beta_{\xi} h^{3}+\beta_{\xi_{5}} h^{5}\right)=-L \\
& S_{\alpha} \ddot{h}+I_{\alpha} \ddot{\alpha}+C_{\alpha} \dot{\alpha}+K_{\alpha}\left(\alpha+\beta_{\alpha} \alpha^{3}+\beta_{\alpha_{5}} \alpha^{5}\right)=M
\end{aligned}
$$

with the structural nonlinearity approximated in a polynomial form. ${ }^{13}$ The lift, $L$, is defined positive upward according to the usual sign convention in aerodynamics. The plunge displacement, $h$, is positive downward, as it is conventionally done in aeroelasticity. In nondimensional form, the equations of motion become

$$
\begin{aligned}
& \xi^{\prime \prime}+x_{\alpha} \alpha^{\prime \prime}+2 \zeta_{\xi} \frac{\bar{\omega}}{U^{*}} \xi^{\prime}+\left(\frac{\bar{\omega}}{U^{*}}\right)^{2}\left(\xi+\beta_{\xi} \xi^{3}+\beta_{\xi_{5}} \xi^{5}\right)=-\frac{1}{\pi \mu} C_{L}(\tau) \\
& \frac{x_{\alpha}}{r_{a}^{2}} \xi^{\prime \prime}+\alpha^{\prime \prime}+2 \zeta_{\alpha} \frac{1}{U^{*}} \alpha^{\prime}+\left(\frac{1}{U^{*}}\right)^{2}\left(\alpha+\beta_{\alpha} \alpha^{3}+\beta_{\alpha_{5}} \alpha^{5}\right)=\frac{2}{\pi \mu r_{a}^{2}} C_{m}(\tau)
\end{aligned}
$$

where nondimensional parameters are defined in the nomenclature. Differentiation with respect to $t$, indicated by $\left(\dot{)}\right.$, is replaced by a differentiation with respect to $\tau, \dot{()}=U / b()^{\prime}$.

The aerodynamics is given by modelling an incompressible two-dimensional flow as in Theodorsen. ${ }^{14}$ The total aerodynamic loads consist of contributions arising from the aerofoil motion, flap deflection and the penetration into a gusty field

$$
C_{L}(\tau)=C_{L}^{a}(\tau)+C_{L}^{\delta}(\tau)+C_{L}^{g}(\tau), \quad C_{m}(\tau)=C_{m}^{a}(\tau)+C_{m}^{\delta}(\tau)+C_{m}^{g}(\tau)
$$

The generalization of the aerodynamic loads to an arbitrary input time-history is obtained through convolution or Duhamel integral. For a practical evaluation of the Duhamel integral, an exponential approximation is used for the Wagner ${ }^{15}$ and Küssner ${ }^{16}$ functions which describe, respectively, the indicial build-up of the circulatory part of the lift and the lift build-up for the penetration into a sharp-edged gust. This implies that the governing equations in Eqs. (38) and (39) are a set of integro-differential equations (IDEs) for which analytical solutions are difficult to obtain. In addition, most of the methods to study nonlinear systems are developed for ODEs. The mathematical procedure based on defining additional variables and equations describing their evolution is used to convert the set of IDEs into a set of ODEs. The procedure in Lee et 
al. ${ }^{17}$ is similarly applied to the manipulation of the contributions associated with the flap rotation and the gust perturbation. Then, the equations of motion are written in the form of Eq. (2), where

$$
\boldsymbol{w}=\left[\alpha, \xi, \alpha^{\prime}, \xi^{\prime}, w_{5}, w_{6}, w_{7}, w_{8}, w_{9}, w_{10}, w_{11}, w_{12}\right]^{T}, \quad \boldsymbol{u}_{c}=\left[\delta, \delta^{\prime}, \delta^{\prime \prime}\right]^{T}, \quad \boldsymbol{u}_{d}=W_{g}
$$

By defining 8 auxiliary variables for the aerodynamic states, the state-space vector has dimension 12 . The zeroth and first order control input derivatives are dependent upon the flap rotational acceleration, and thus incorporated into the state-space vector in the control problem formulation. The residual vector, $\boldsymbol{R}$, is a nonlinear function of $\boldsymbol{w}$ when structural nonlinearities are modelled.

The system derived from coupling the pitch-plunge aerofoil structural model with two-dimensional potential flow is a model problem to test the methods. The generation of linear and nonlinear ROMs and their use to design a control law are now presented.

\section{A. Linear Stability Analysis}

Methods for locating the bifurcation point can be divided into two classes. For indirect methods, a bifurcation point is calculated solving Eq. (2) repeatedly for different values of the bifurcation parameter and detecting a change of sign of a test function which classifies the bifurcation point. For the Hopf bifurcation, one possible test is to calculate all the eigenvalues of the large-order system and see when one pair crosses the real axis. When the crossing has been detected, the secant method can be used to solve for a zero real part of the eigenvalue. The direct methods solve the system of equations in Eq. (2) augmented by additional equations that characterize the bifurcation point. In this study, the indirect method was used. The small size of the model problem allows to use standard routines to solve the eigenvalue problem.

To validate the prediction of the linear instability point with data available in the literature, the aeroelastic parameters shown in Table 1 were used. The nonlinear spring constants are nil. The traces of the critical

\begin{tabular}{cc}
\hline \hline Parameter & Value \\
\hline $\bar{\omega}$ & 0.2 \\
$\mu$ & 100.0 \\
$a_{h}$ & -0.5 \\
$x_{\alpha}$ & 0.25 \\
$r_{\alpha}$ & 0.5 \\
$\zeta_{\alpha}$ & 0.0 \\
$\zeta_{\xi}$ & 0.0 \\
\hline \hline
\end{tabular}

Table 1. Reference values of the pitch-plunge aerofoil model for linear stability analysis

complex eigenvalue as the reduced velocity $U^{*}$ is changed are shown in Fig. 2(a). The crossing happens for $U_{L}^{*}=6.285$, which is the same value reported by Liu et al. ${ }^{18}$ For the dynamic aeroelastic results presented in the following sections, the freestream speed is $U^{*}=0.95 U_{L}^{*}$ and the corresponding root locus is illustrated in Fig. 2(b). There are two pairs of complex conjugate eigenvalues and eight real eigenvalues with negative real part. Repeated eigenvalues $(\lambda=-0.0455$ and -0.3$)$ correspond to the constants in the exponential approximation of the Wagner function. Two eigenvalues, $\lambda=-0.1393$ and -1.802 , correspond to the constants in the exponential approximation of the Küssner function.

\section{B. Open-Loop Dynamic Aeroelastic Response to Gust}

Several tests were made to assess the accuracy of the ROM in the prediction of the dynamic response to a gust perturbation. Approaches to represent gust perturbations include discrete and continuous models. ${ }^{19}$ Examples of the former are the "one-minus-cosine" and sinus functions, and examples of the latter are the power spectra density of Dryden and Von Karman models. However, to focus attention on a particular case, a deterministic sinusoidal gust of the form

$$
W_{g}(\tau)=W_{0} \sin \left(\frac{2 \pi}{h_{g}}\left(\tau-\tau_{0}\right)\right) \quad \text { for } \tau_{0} \leq \tau \leq \tau_{0}+h_{g} n_{c}
$$




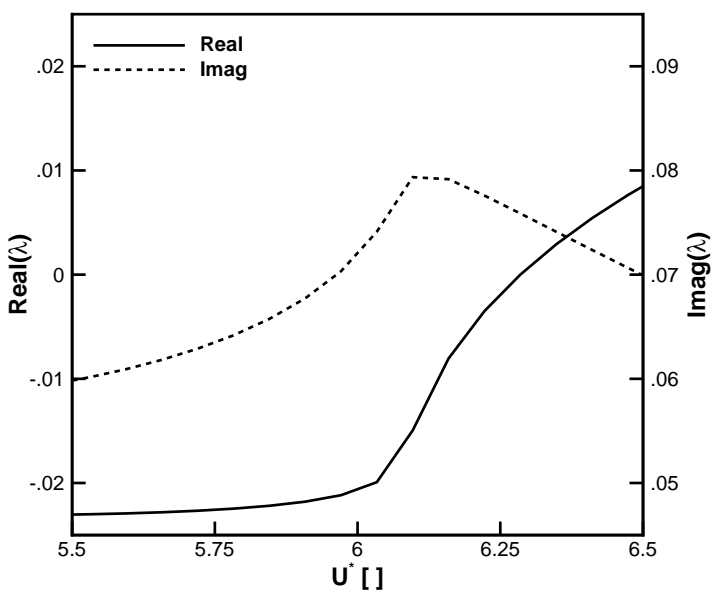

(a) Critical mode traces $\left(U_{L}^{*}=6.285\right)$

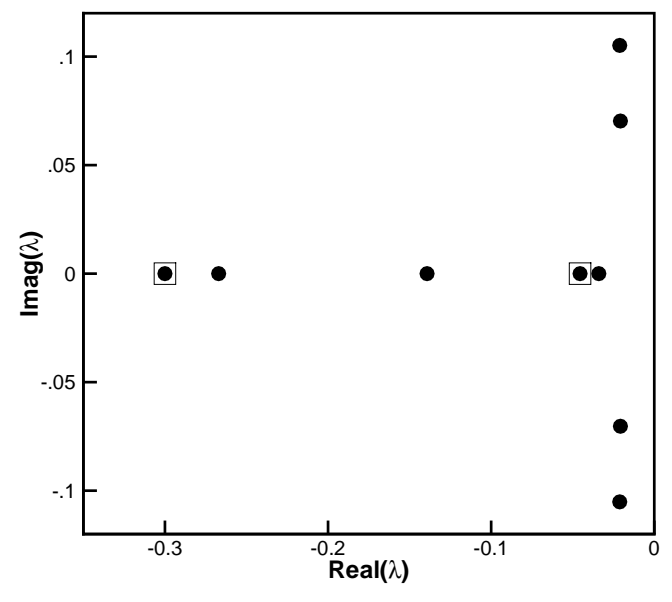

(b) Root locus near the origin

Figure 2. Pitch-plunge aerofoil using incompressible two-dimensional aerodynamics; (a) critical mode traces; (b) root locus at $U^{*} / U_{L}^{*}=0.95$, real eigenvalue $\lambda=-1.802$ is not included in the plot

was used. The choice of a discrete gust reflects a practical question, but it does not represent a fundamental problem in the phase of model reduction. In fact, the gust is an external excitation and its particular form is irrelevant when reducing the dynamics of the full-order model. More realistic gust models will be considered in verifying the control law. One sinusoidal cycle was simulated, $n_{c}=1$. Several gusts of different wavelength were selected with the corresponding reduced frequency spanning the spectrum of the system eigenvalues.

It is possible to compare the open-loop dynamic aeroelastic response obtained integrating the FOM with the response predicted using ROMs. This allows to investigate the convergence of the reduced model for increasing number of modes in approximating the full system dynamics. Results are presented for an aerofoil section with and without structural nonlinearities.

Figure 3 shows the time-history of the angle of attack and plunge displacement in response to a gust of wavelength $h_{g}=20$ and intensity $W_{0}=0.001$. In this first case, the structural nonlinearities are neglected. Note that the gust shape is shown in the lower part of the same figure for a comparison with the dominant wavelength of the response. The ROM was enhanced by adding the least damped eigenvalue and the corresponding eigenvector not already included in the existing ROM. The inclusion of the first structural mode is not sufficient to get a response representative of the FOM. An improvement in the ROM solution is achieved when including the second structural mode. This ROM predicts well the free response of the system after the gust moves away from the aerofoil but differences between ROM and FOM are observed when the aerofoil penetrates into the gust. No improvement in the ROM response was found by adding the subsequent three modes, and these are not plotted. The ROM with the sixth mode $(\lambda=-0.1393)$ reproduces the FOM response for all times, suggesting that the sixth mode couples the structural response with the gust input. The reason is that the eigenvalue corresponds to the slower time constant in the approximation used for the Küssner function. Based on these results, unwanted effects may be experienced when a control law formulated on the basis of the one-mode ROM is applied to control the FOM.

Tests were made comparing the time-response of the FOM with and without cubic stiffness in pitch. For increasing intensity of the gust perturbation, increasing effects of the structural nonlinearities in the dynamic response were found. In the case of the nonlinear structural model here considered, $\beta_{\alpha}=3.0$. Results in Fig. 4 are for a sinusoidal gust of $h_{g}=40.0$ and $W_{0}=0.10$. Note that the gust vertical velocity is one hundred times larger than in the linear case above. As the aerofoil travels into the gusty field, the dynamic responses of the FOM with and without nonlinearities are identical. The motion variables are the largest in magnitude some time after the gust has left the aerofoil, but large differences between FOM and NFOM are also observed in the free-response. The reason for a large overshoot in the response of the linear system is that the nonlinearity is representative of an hardening spring $\left(\beta_{\alpha}>0\right)$. The point here is to illustrate a test case in which the time-response of the FOM is affected by the degree of structural nonlinearity and to 


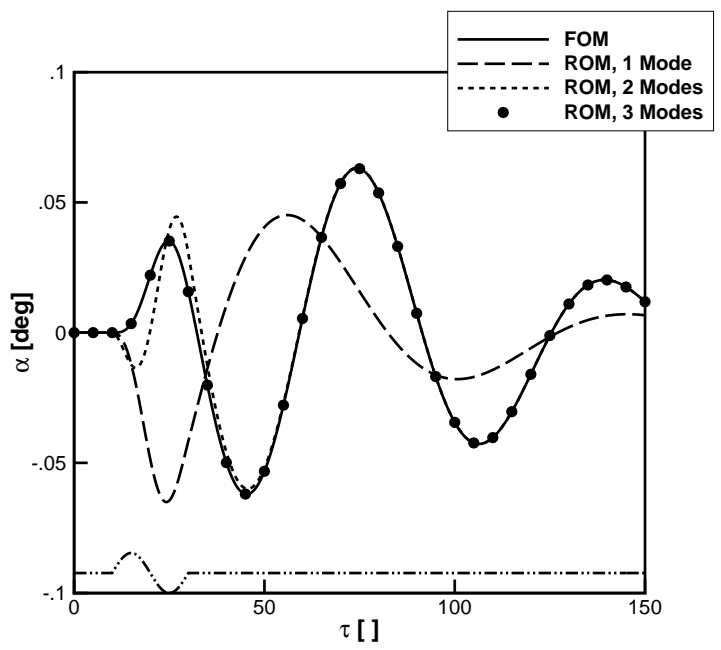

(a) Angle of attack, $\alpha$

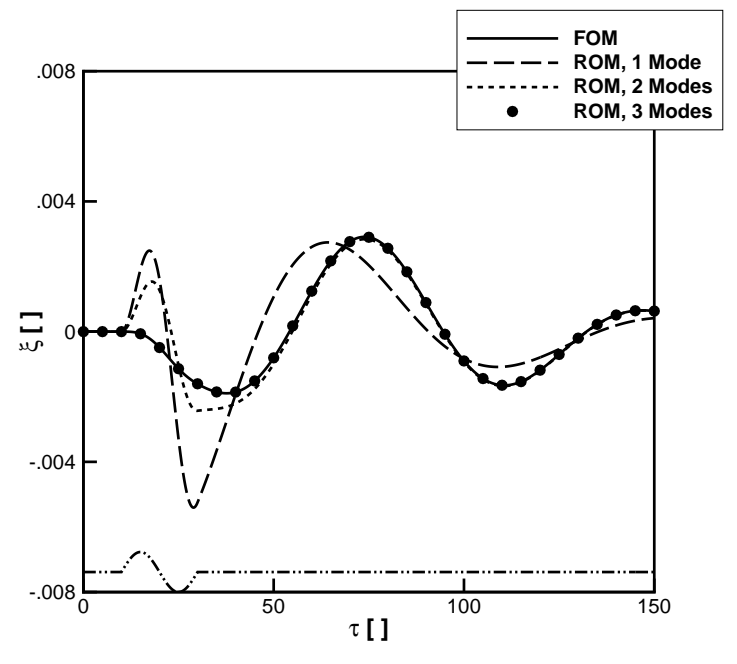

(b) Plunge, $\xi$

Figure 3. Dynamic aeroelastic response to a sinusoidal gust $\left(h_{g}=20.0, W_{0}=0.001\right)$ at $U^{*} / U_{L}^{*}=0.95$ with linear stiffness parameters

demonstrate the nonlinear model reduction in this case.

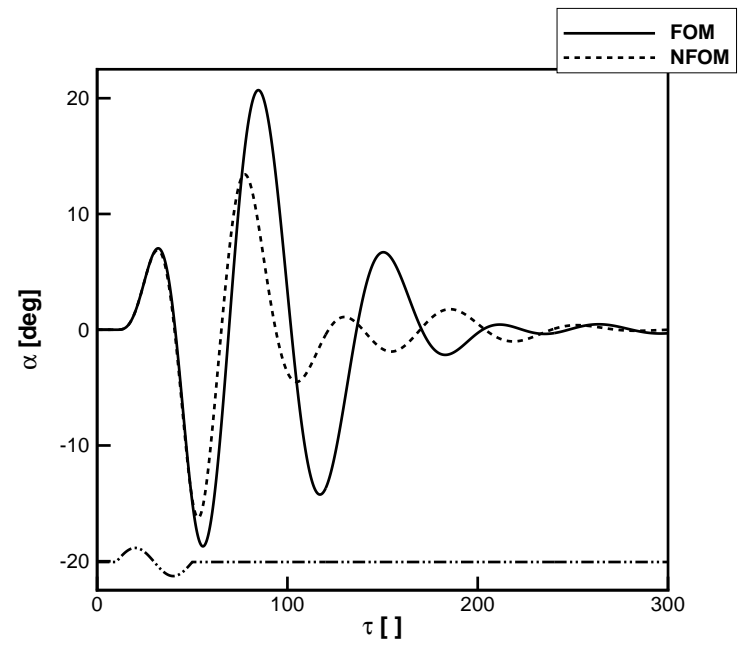

(a) Angle of attack, $\alpha$

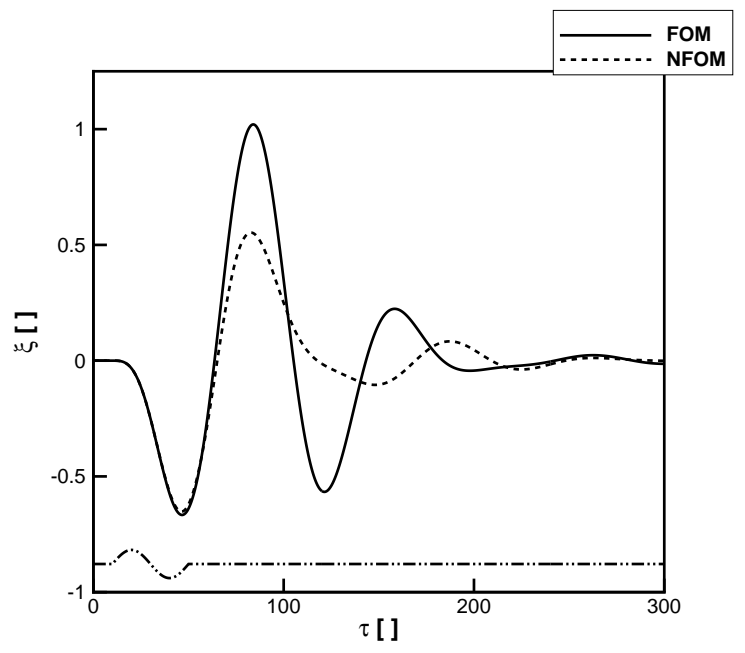

(b) Plunge, $\xi$

Figure 4. Dynamic aeroelastic response of the FOM to a sinusoidal gust $\left(h_{g}=40.0, W_{0}=0.10\right)$ at $U^{*} / U_{L}^{*}=0.95$ with linear and nonlinear $\left(\beta_{\alpha}=3.0\right)$ stiffness parameters

Next, a NROM was created with nonlinear terms up to third order. Several modes were recursively included as described above, and results are shown in Fig. 5. The one-mode NROM response diverged as the gust moves away from the aerofoil with a continuously decreasing angle of attack, and is not plotted. The two-mode prediction provides a reasonable approximation of the reference solution, with a larger deviation occurring when travelling into the gust. The tree-mode NROM prediction is identical to the reference solution by adding the real-valued eigenvalue $\lambda=-0.1393$. A consideration is that the NROM proved adequate to predict the nonlinear response in a case in which a linear FOM has shown large deviations from the nonlinear counterpart (recall Fig. 4). It also demonstrates the improvement achieved in the ROM when including higher-order terms. 


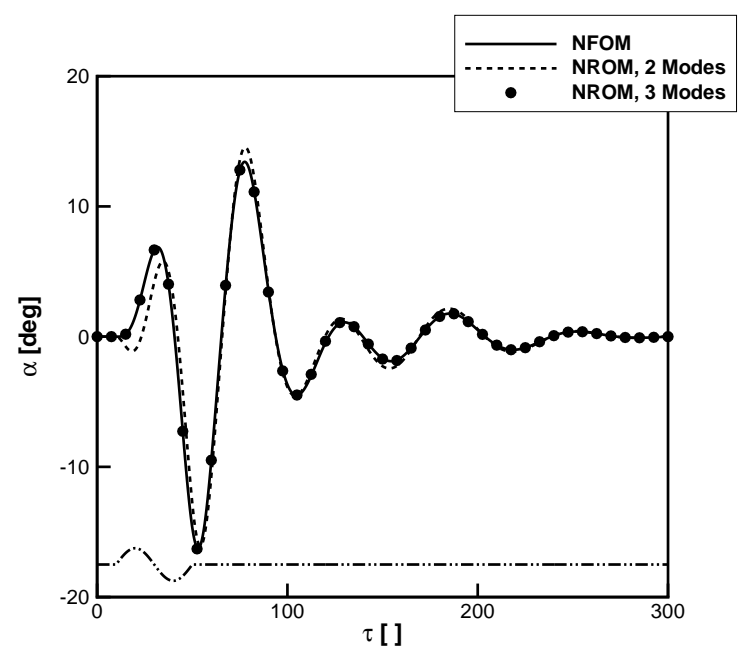

(a) Angle of attack, $\alpha$

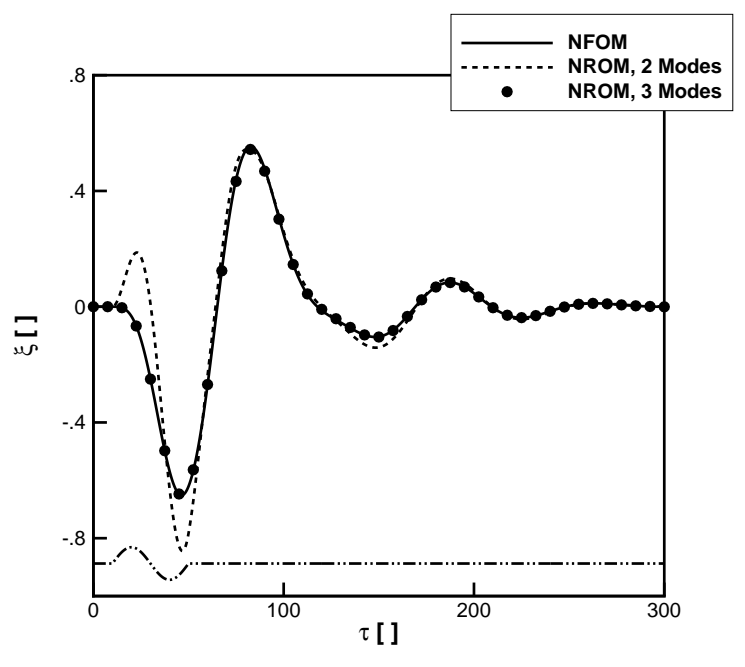

(b) Plunge, $\xi$

Figure 5. Dynamic aeroelastic response to a sinusoidal gust $\left(h_{g}=40.0, W_{0}=0.10\right)$ at $U^{*} / U_{L}^{*}=0.95$ with nonlinear $\left(\beta_{\alpha}=3.0\right)$ stiffness parameters

\section{Closed-Loop Dynamic Aeroelastic Response to Gust}

Tests were made to observe the effect of a linear $\boldsymbol{H}^{\infty}$ controller on the closed-loop dynamic response to a gust perturbation. The controller designed on the basis of a linear ROM is applied, by Eq. (37), to the nonlinear ROM with the aim of achieving gust rejection. The disturbance input to the nonlinear system dynamics is taken as the sinusoidal gust load in Eq. (44) with $h_{g}=20.0$ and $W_{0}=0.10$.

Figure 6(a) shows the closed loop response of the nonlinear reduced-order model, for varying control weights $k_{c}$, plotted against the open loop responses. It can be seen that a small control weight $k_{c}$ produces a controller that is better at suppressing the sinusoidal gust. Conversely, a more significant weight placed on the input $k_{c}$ slows down the variation of $\delta$, as shown in Fig. 6(b), since a stronger weight is placed on $\boldsymbol{u}=\delta^{\prime \prime}$ relative to the system output. Varying the measurement noise weight $k_{d}$ also has an effect on the controller dynamics. A less confident measurement significantly reduces the control input, as illustrated in Figs. 6(c) and 6(d).

We note that the difference between the closed-loop response of linear ROM and the closed loop response of the nonlinear ROM is minimal. This suggests that the $\boldsymbol{H}^{\infty}$ controller, which was designed on the linearised system dynamics, is able to effectively suppress gust disturbances to the nonlinear reduced order model. A possible explanation for this desirable behaviour is that the nonlinearity has a cubic hardening effect, which itself serves to stabilise the system.

\section{Pitch-Plunge Aerofoil using Computational Fluid Dynamics}

The coupled system is derived from coupling the structural model for an aerofoil free to move in pitch and plunge with a CFD solver. The CFD solver used is the Parallel Meshless (PML) solver of the University of Liverpool which solves the Euler, laminar and Reynolds-averaged Navier-Stokes equations on clouds of points, as opposed to cells of a finite volume grid. The basic approach of the solver is summarised in Ref., ${ }^{20}$ the main features of which are the use of an implicit meshless scheme to simulate complex flows around multibody configurations in relative motion, a preconditioned Krylov subspace method to perform the integration in time, and the use of a combination of approximate, analytical Jacobian matrices and an inexact linear system solver to improve the computational efficiency of the scheme.

Two challenges arise when using CFD as the source of the aerodynamic predictions. The solution of a large sparse linear system arising from an eigenvalue problem is needed for model generation. Then, the ability to simulate a gust encounter is a prerequisite to calculate the gust influence coefficients for model generation. 


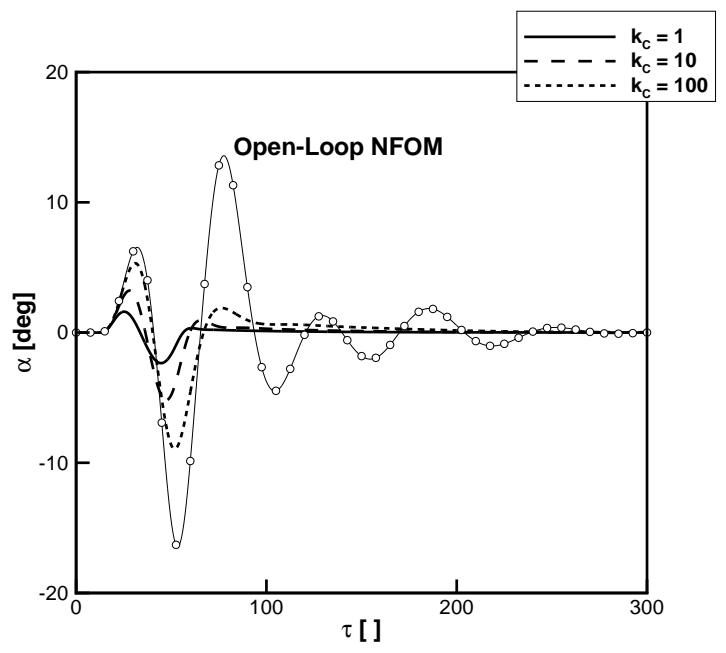

(a) Angle of attack, $\alpha$

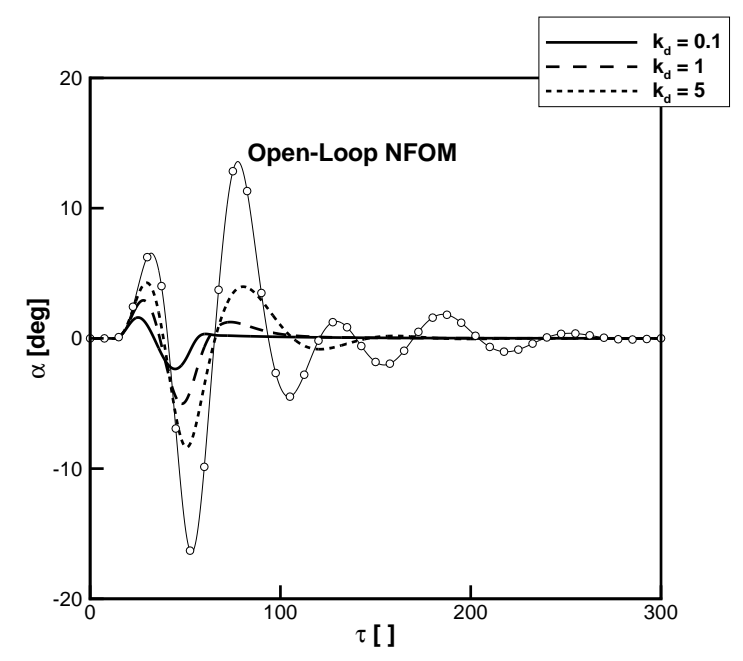

(c) Angle of attack, $\alpha$

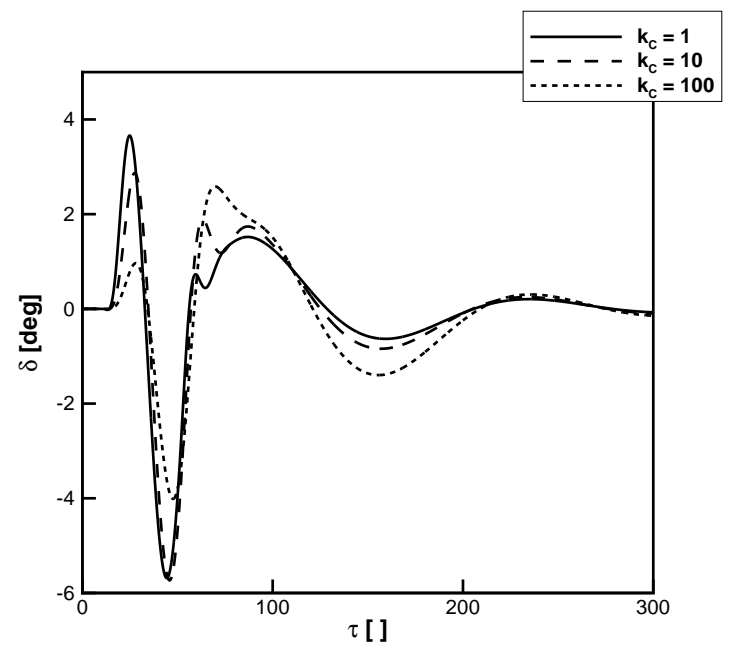

(b) Flap deflection, $\delta$

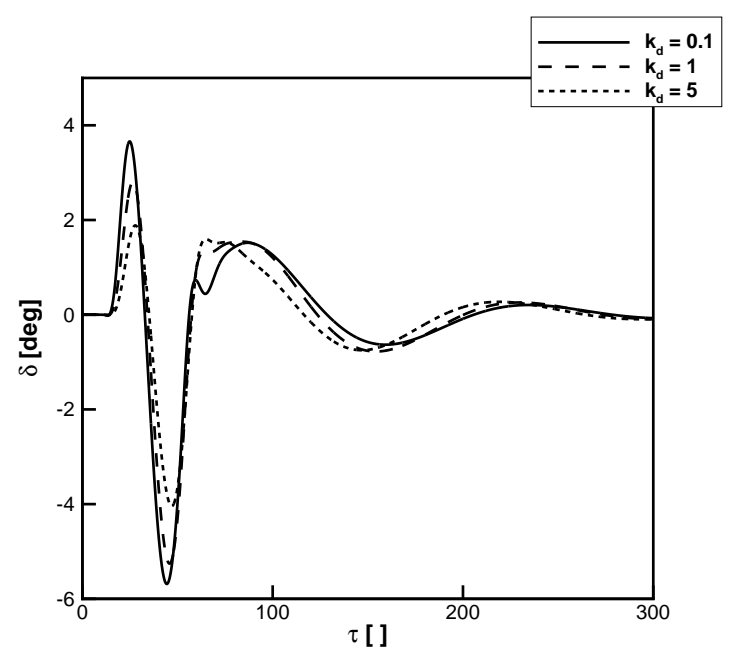

(d) Flap deflection, $\delta$

Figure 6. Dependencies of angle of attack and flap deflection time-histories on control weights; (a)-(b) varying $k_{c}$ with $k_{d}=0.1$; (c)-(d) varying $k_{d}$ with $k_{c}=1$ 
To overcome the first problem, the Schur complement eigenvalue formulation is used. The method leads to a small nonlinear eigenvalue problem that can be solved rapidly by removing the need to solve large sparse linear systems that are almost singular. The coupled system Jacobian matrix of Eq. (2) is most conveniently done by partitioning the matrix as

$$
\boldsymbol{A}=\left[\begin{array}{ll}
\frac{\partial \boldsymbol{R}_{f}}{\partial \boldsymbol{w}_{f}} & \frac{\partial \boldsymbol{R}_{f}}{\partial \boldsymbol{w}_{s}} \\
\frac{\partial \boldsymbol{R}_{s}}{\partial \boldsymbol{w}_{f}} & \frac{\partial \boldsymbol{R}_{s}}{\partial \boldsymbol{w}_{s}}
\end{array}\right]=\left[\begin{array}{ll}
\boldsymbol{A}_{f f} & \boldsymbol{A}_{f s} \\
\boldsymbol{A}_{s f} & \boldsymbol{A}_{s s}
\end{array}\right]
$$

The block $\boldsymbol{A}_{f f}$ represents the influence of the fluid unknowns on the fluid residual and has by far the largest number of nonzeros for the pitch-plunge aerofoil. The term $\boldsymbol{A}_{f s}$ arises from the dependence of the CFD residual on the mesh motion and speeds, which depend in turn on the structural solution, and is evaluated by finite difference. The term $\boldsymbol{A}_{s f}$ is due to the dependence of the generalized forces on the surface pressures. Finally, the block $\boldsymbol{A}_{s s}$ is the Jacobian of the structural equations with respect to the structural unknowns.

Write the coupled system eigenvalue problem as

$$
\left[\begin{array}{ll}
\boldsymbol{A}_{f f} & \boldsymbol{A}_{f s} \\
\boldsymbol{A}_{s f} & \boldsymbol{A}_{s s}
\end{array}\right] \boldsymbol{p}=\lambda \boldsymbol{p}
$$

where $\boldsymbol{p}$ and $\lambda$ are the complex eigenvector and eigenvalue, respectively. Partition the eigenvector as

$$
\boldsymbol{p}=\left[\boldsymbol{p}_{f}^{T}, \boldsymbol{p}_{s}^{T}\right]^{T}
$$

By substituting $\boldsymbol{p}_{f}$ from the first set of equations into the second set of equations in Eq. (46), it can be found that the eigenvalue $\lambda$, assuming it is not an eigenvalue of $\boldsymbol{A}_{f f}$, satisfies the nonlinear eigenvalue problem

$$
\boldsymbol{S}(\lambda) \boldsymbol{p}_{s}=\lambda \boldsymbol{p}_{s}
$$

where $\boldsymbol{S}(\lambda)=\boldsymbol{A}_{s s}-\boldsymbol{A}_{s f}\left(\boldsymbol{A}_{f f}-\lambda I\right)^{-1} \boldsymbol{A}_{f s}$. The matrix $\boldsymbol{S}(\lambda)$ is the sum of the structural matrix and a second term arising from the coupling of the fluid and structure. The nonlinear Eq. (48) is solved using Newton's method. To overcome the cost of forming the residual and its Jacobian matrix at each iteration, a Taylor expansion of $\left(\boldsymbol{A}_{f f}-\lambda I\right)^{-1}$ is used. More details on the Schur complement eigenvalue solver and its application to realistically sized aeroelastic models can be found in Ref. ${ }^{21}$

The second problem to address is the simulation of gust loads using a CFD solver. Based on previous work, ${ }^{22}$ the approach in Ref. ${ }^{23}$ referred to as the field-velocity approach is used. The gust is introduced into an existing CFD solver by modification of the velocity of grid points during the unsteady motion of the aerofoil. A disadvantage of the field-velocity approach is that the gust is assumed frozen, and the influence of the structural response on the gust is neglected. Thus, the generality of CFD is not fully exploited. The approach has received widespread use because of the lack of alternative methods.

To form the gust influence coefficients in the ROM state-space formulation, the last term in Eq. (13) needs to be computed. Denote by $u_{g, z}$ the vertical component of gust velocity. Two methods have been tested. The first consists of perturbing the velocity of all grid points at the same time, and the term $\frac{\partial \boldsymbol{R}}{\partial u_{g, z}}$ is computed by finite difference with two residual evaluations. An alternative method is to perturb the velocity of each grid point in turn, and calculate by finite difference the columns of a large sparse matrix, $\boldsymbol{B}_{g}$. The columns are calculated as

$$
\boldsymbol{B}_{g, i}=\frac{\partial \boldsymbol{R}}{\partial u_{g, z}^{(i)}} \quad \text { for } i=1, \ldots, n_{p}
$$

where $n_{p}$ is the total number of grid points and $u_{g, z}^{(i)}$ the perturbation of the vertical velocity applied to the $i$-th grid point. The action of this matrix on a time-dependent vector, which depends on the shape of the gust and location of the grid points, is needed. Finally, the last term in Eq. (13) is rewritten as

$$
\overline{\boldsymbol{\psi}}_{i}^{T} \boldsymbol{B}_{g} \frac{\partial \boldsymbol{u}_{g, z}}{\partial W_{g}}
$$

The first two terms on the left involve a matrix-vector multiplication, and this can be done when forming the ROM. At every time step iteration, the vector on the right has to be updated and the scalar product of two vectors evaluated. 
The test problem considered herein is for the NACA 0012 aerofoil at zero incidence. The parameters for the structural model are given in Table 2. The testcase corresponds to the heavy case described in Ref. ${ }^{24}$ The point distribution used for the Euler calculations is shown in Fig. 7 and consists of 7974 grid points. A refinement study can be found in Ref. ${ }^{20}$

\begin{tabular}{cc}
\hline \hline Parameter & Value \\
\hline $\bar{\omega}$ & 0.343 \\
$\mu$ & 100.0 \\
$a_{h}$ & -0.2 \\
$x_{\alpha}$ & 0.2 \\
$r_{\alpha}$ & 0.539 \\
$\zeta_{\alpha}$ & 0.0 \\
$\zeta_{\xi}$ & 0.0 \\
\hline \hline
\end{tabular}

Table 2. Reference values of the pitch-plunge aerofoil model

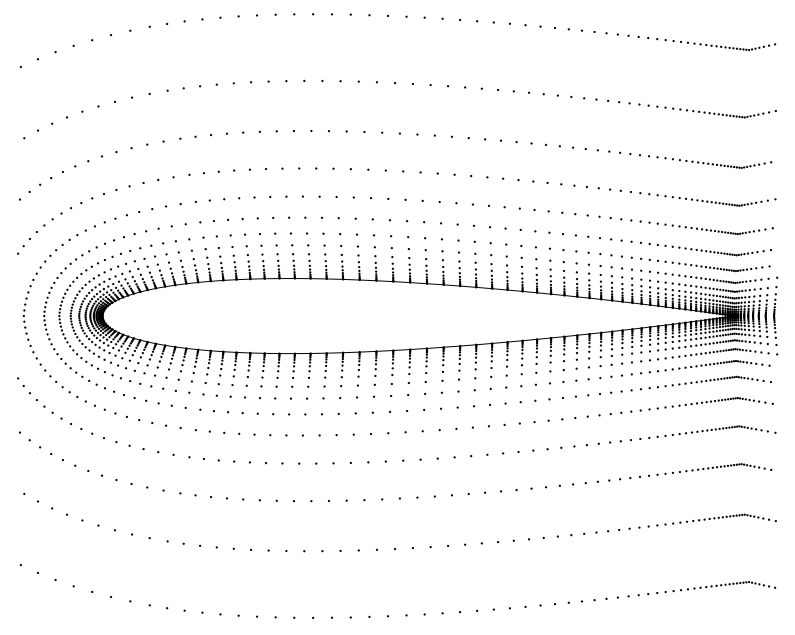

Figure 7. Point distribution for NACA 0012 aerofoil

\section{A. Free-Response to Initial Conditions}

At Mach $M=0.6$, the aerofoil in the heavy configuration becomes unstable for a reduced velocity larger than 4 . For the results presented, $U^{*}=2.0$ and the system is asymptotically stable. A linear reduced model was formed using the Schur complement tracking the two structural normal modes. Hence, the ROM has dimension 2 .

The free-response to an initial angle of attack $\alpha_{0}=1 \mathrm{deg}$ is illustrated in Fig. 8. The time-accurate simulation of the coupled system was performed over 2000 time steps, and is denoted in figure by "FOM". The predictions obtained using a linear ROM are in excellent agreement. The cost for ROM generation was a fraction of the cost incurred by the time-accurate simulation.

\section{B. Open-Loop Dynamic Aeroelastic Response to Gust}

Fundamental to this work is the simulation of a gust encounter. To test the model reduction, two testcases are presented for various gust shapes. The first case features a step-change in angle of attack, which is representative of a gust of constant intensity instantaneously affecting the entire flow domain. The second case is for a discrete "1-cos" gust which is convected downstream at freestream speed. To allow a comparison 


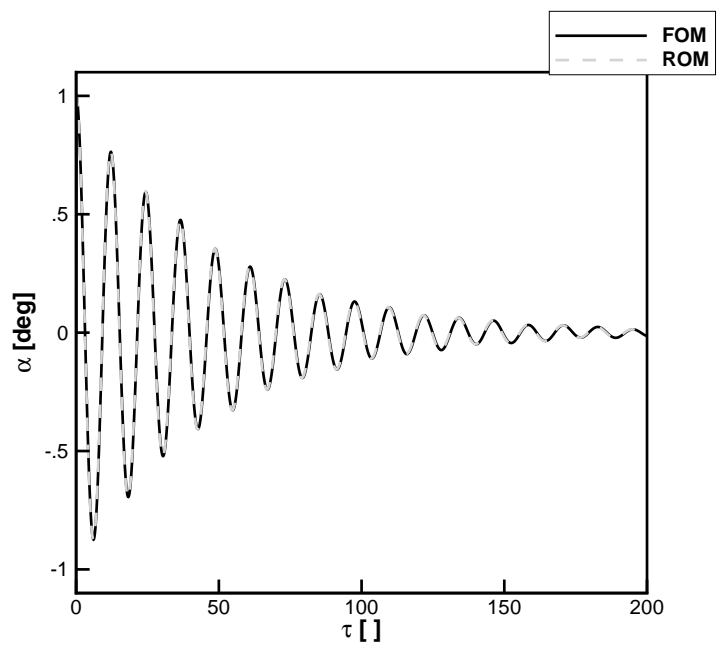

(a) Angle of attack, $\alpha$

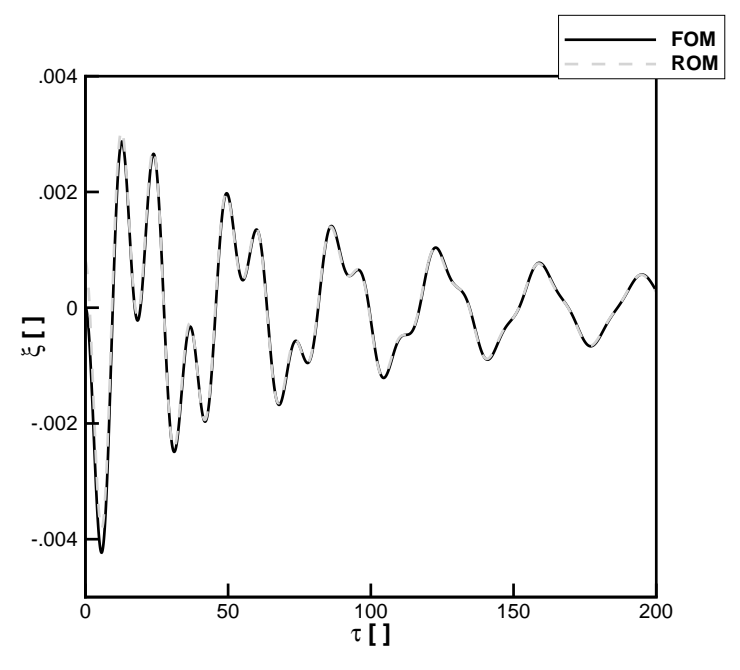

(b) Plunge, $\xi$

Figure 8. Free-response of pitch-plunge aerofoil for initial condition $\alpha_{0}=1 \mathrm{deg}$, Mach number $M=0.6$ and reduced velocity $U^{*}=2.0$; the term "FOM" indicates the fully coupled system using CFD

of CFD-based results with those based on potential flow, the Mach number was set to 0.3. As before, the ROM was generated with 2 modes.

Figure 9 shows the time-history of the angle of attack and plunge displacement in response to a stepchange in angle of attack. For a gust intensity of $W_{0}=0.01$, the steady-state increment in angle of attack is $\Delta \alpha=0.57 \mathrm{deg}$. Results obtained using CFD and potential theory are reported, respectively, on the upper and lower part of the figure. Because of the benign flow conditions, the similarity in the dynamic response of FOMs is not unexpected. The ROM predictions are qualitatively similar to their FOM counterparts. The reason for a constant offset between each pair of results is that 2 modes are inadequate to recover steady-state effects. To overcome this, more modes are needed. An alternative is to use the mode acceleration method, which consists in complementing the solution with the missing terms from the modal expansion of the static response. Note that any realistic gust has a finite duration, and this case served for the purpose of testing the model reduction.

The response to a discrete "1-cos" gust is examined in Fig. 10. The point here is to illustrate the effect of the approximations introduced when calculating the gust terms in the ROM state-space formulation. The gust has the form

$$
W_{g}(\tau)=\frac{W_{0}}{2}\left(1-\cos \left(\frac{2 \pi}{h_{g}}\left(\tau-\tau_{0}\right)\right)\right) \quad \text { for } \tau_{0} \leq \tau \leq \tau_{0}+h_{g} n_{c}
$$

where $h_{g}=12.5, W_{0}=0.01$, and $n_{c}=1$. The gust perturbation is restricted to within 6.25 aerofoil chords, and the remaining domain is unaffected by the gust. At the initial time, the gust front is located at the leading edge of the aerofoil. The gust travels downstream at freestream speed and at $\tau=2$ the gust front is at the trailing edge of the aerofoil. Two ROM predictions are compared in figure and differ only in the computation of gust influence coefficients. For the first, referred to as "ROM (vector)" in figure, the gust terms were obtained by perturbing the velocity of all grid points at the same time. For the curve denoted by "ROM (matrix)", the sparse matrix $\boldsymbol{B}_{g}$, with columns defining the dependence of the residual on the velocity of each grid point in turn, was generated. In the case of a gust varying in space and time, the approximation introduced using the first approach leads to poor results compared to the FOM. On the opposite, the matrix approach agrees well with the FOM. Note that the small deviations for small times are attributable to using two modes only, and a similar feature was observed in Fig. 3. The cost of model generation was neglegible with respect to the cost incurred by the time-accurate simulation. Once created, the ROM can be used at no additional cost in a parametric study, for example, in determining the worst-case gust loads. 


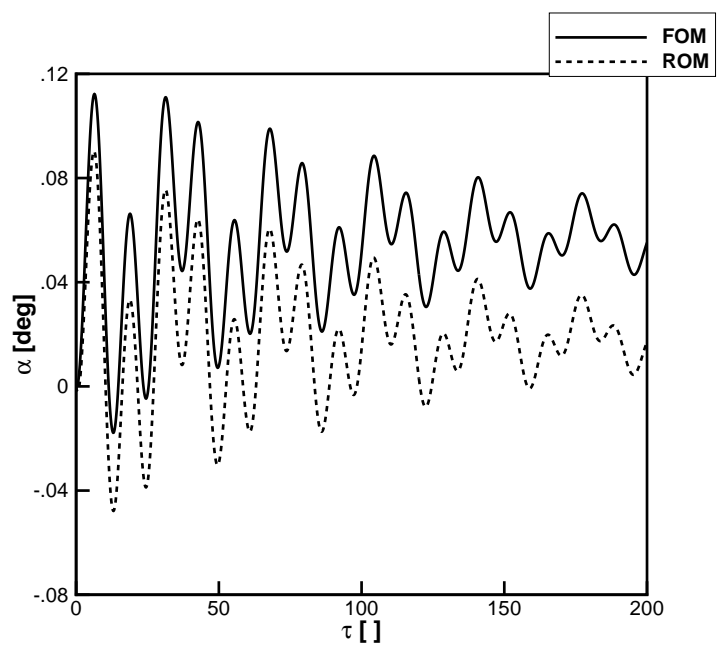

(a) Angle of attack, $\alpha$ (CFD)

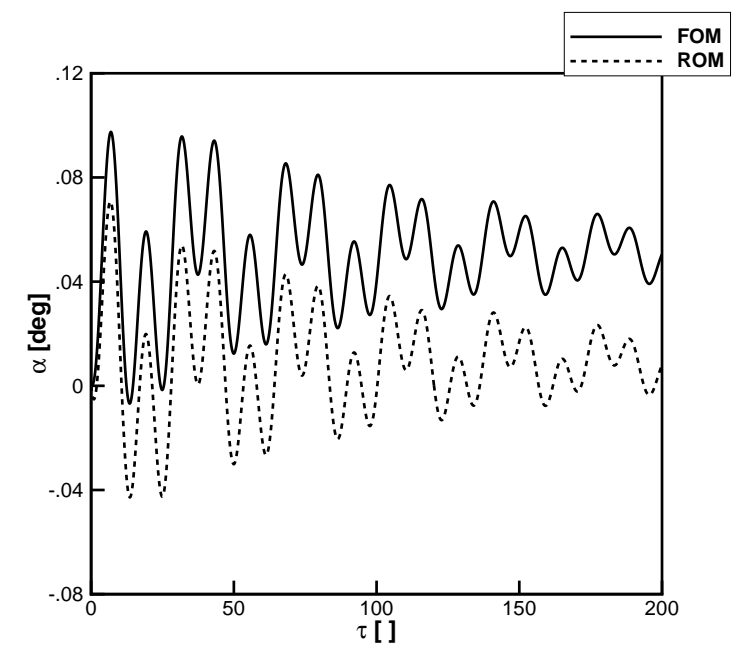

(c) Angle of attack, $\alpha$ (potential theory)

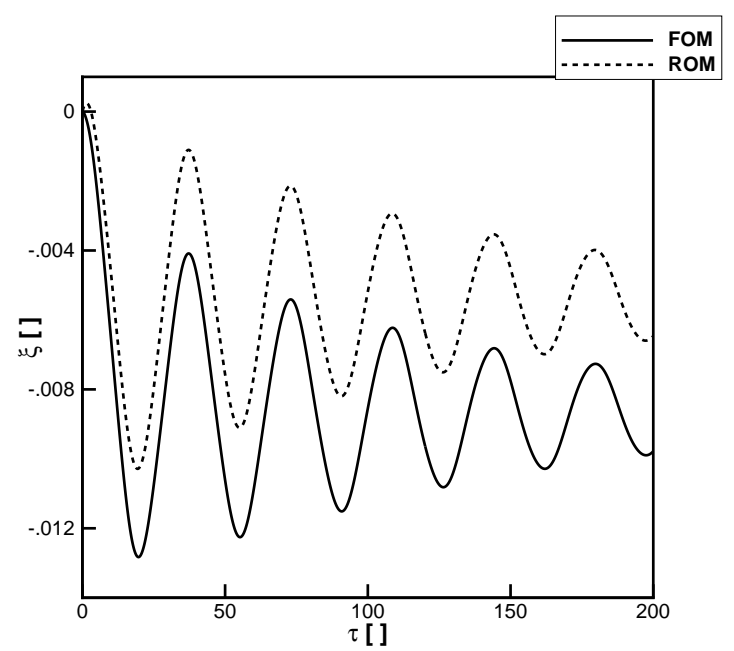

(b) Plunge, $\xi(\mathrm{CFD})$

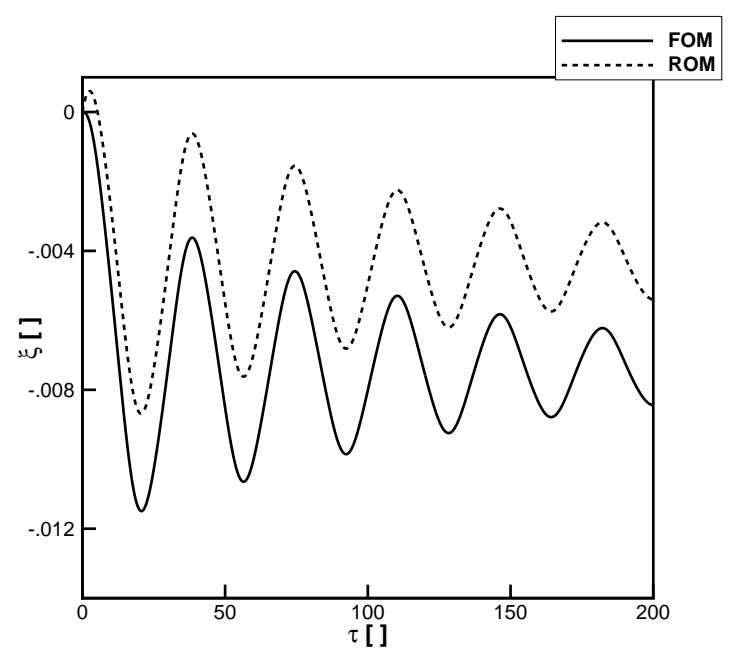

(d) Plunge, $\xi$ (potential theory)

Figure 9. Response of pitch-plunge aerofoil section to a step-change in angle of attack $(\Delta \alpha=0.57 \mathrm{deg})$, Mach number $M=0.3$, and reduced frequency $U^{*}=2 ;(\mathbf{a})-(\mathbf{b})$ the term "FOM" indicates the fully coupled system using CFD; (c)-(d) the term "FOM" indicates the fully coupled system using potential two-dimensional theory 


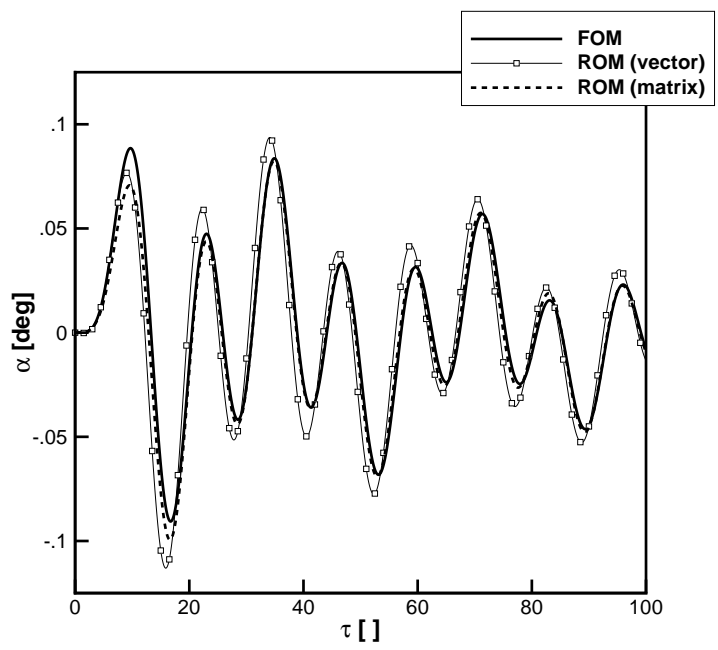

(a) Angle of attack, $\alpha$

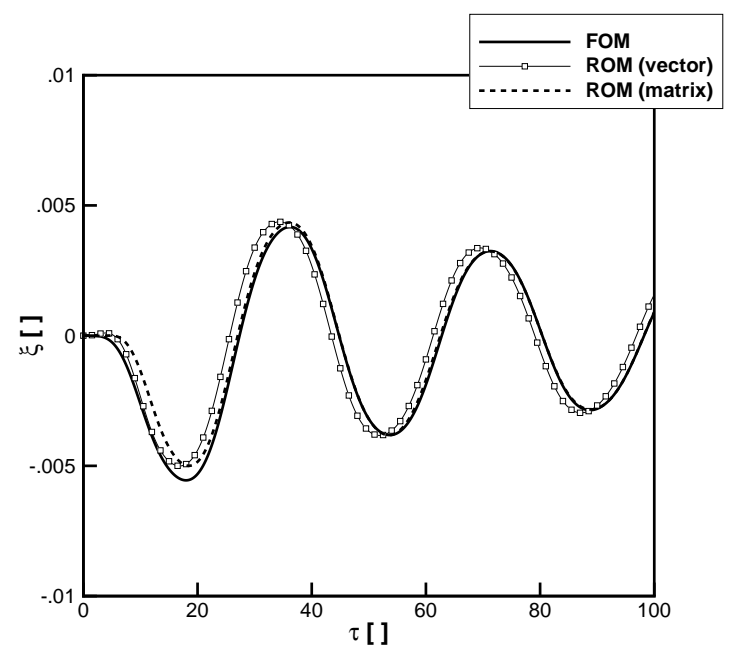

(b) Plunge, $\xi$

Figure 10. Response of pitch-plunge aerofoil section to a "1-cos" gust ( $W_{0}=0.01$ and $\left.h_{g}=12.5\right)$, Mach number $M=0.3$, and reduced frequency $U^{*}=2$; the term "FOM" indicates the fully coupled system using CFD

\section{Geometrically-Exact Nonlinear Beam}

For the structural model, the geometrically-exact nonlinear beam equations are used. ${ }^{25}$ Results are obtained using two-noded displacement-based elements. In a displacement-based formulation, nonlinearities arising from large deformations are cubic terms, as opposed to an intrinsic description where they appear up to second order. The finite element beam code used in this study has been tested extensively, and more details can be found, for example, in Ref. ${ }^{2}$

For the aerodynamic model, the unsteady potential two-dimensional theory is used (see $\S \mathrm{V}$ ). For each aerofoil section, aerodynamic forces are treated as follower forces that depend on the local instantaneous angle of attack of the wing aerofoil.

The problem considered is a wing representative of a flexible HALE aircraft. The wing is modelled as clamped at one side and free at the other. The wing has an aspect ratio of 16 , and the root chord is $1 \mathrm{~m}$. The reference system adopted has the $x$-axis running along the beam span from the root to the tip, the $y$-axis along the chord, and the $z$-axis directed upward.

Data for the geometry, mass and stiffness distribution are available in Ref. ${ }^{26}$ Tests were made to ensure results were independent of the number of elements used, and it was decided that 16 elements were adequate. Results for the structural dynamic model are examined first, and then results for the coupled nonlinear aeroelastic system are presented.

\section{A. On the Significance of Nonlinear Terms}

The nonlinear model reduction in this work is based on a Taylor series expansion around the equilibrium point, $\boldsymbol{w}_{0}$. A consideration is that the series may not converge, or converge to a wrong solution, for a large perturbation, $\Delta \boldsymbol{w}$. The evaluation of higher-order Jacobians may also suffer from truncation and rounding errors, and a range in the step size is needed to ensure the computations are independent of the step size used.

Results presented in Fig. 11 address these two problematics. The equilibrium point is assumed to be the undeformed initial geometry, and the aerodynamics is switched off. A perturbation proportional to the first bending mode was chosen, $\Delta \boldsymbol{w}=\phi_{1} \cdot \eta$, where $\eta$ is the amplification factor. Denote by $\boldsymbol{R}\left(\boldsymbol{w}_{0}+\Delta \boldsymbol{w}\right)$ the nonlinear residual evaluated at the current perturbed configuration. The nonlinear residual was Taylor expanded up to third order using matrix-free products which involve terms like $\boldsymbol{R}\left(\boldsymbol{w}_{0}+\varepsilon \cdot \Delta \boldsymbol{w}\right)$, where $\varepsilon$ is the step size of the finite difference. The $\mathrm{L} 2$ norm of the error between the nonlinear residual evaluation and 
the Taylor expansion was calculated for a range of $\varepsilon$. The notation used in Fig. 11 is as follows

$$
\begin{aligned}
& \text { L2 [1st Order] }=\left\|\boldsymbol{R}\left(\boldsymbol{w}_{0}+\Delta \boldsymbol{w}\right)-\boldsymbol{A} \Delta \boldsymbol{w}\right\|_{2} \\
& \text { L2 [1st Order FD] }=\left\|\boldsymbol{R}\left(\boldsymbol{w}_{0}+\Delta \boldsymbol{w}\right)-(\boldsymbol{A} \Delta \boldsymbol{w})_{F D}\right\|_{2} \\
& \text { L2 [2nd Order }]=\left\|\boldsymbol{R}\left(\boldsymbol{w}_{0}+\Delta \boldsymbol{w}\right)-\boldsymbol{A} \Delta \boldsymbol{w}-1 / 2 \boldsymbol{B}\right\|_{2} \\
& \text { L2 [3rd Order }]=\left\|\boldsymbol{R}\left(\boldsymbol{w}_{0}+\Delta \boldsymbol{w}\right)-\boldsymbol{A} \Delta \boldsymbol{w}-1 / 2 \boldsymbol{B}-1 / 6 \boldsymbol{C}\right\|_{2}
\end{aligned}
$$

Note that the system Jacobian matrix is available analytically, and tests were made to approximate the matrix-vector product by using finite difference, Eq. (53). It was found that the error reduces drastically when nonlinear terms are included in the Taylor expansion, and that matrix-free products can be calculated accurately over a wide range in the step size.

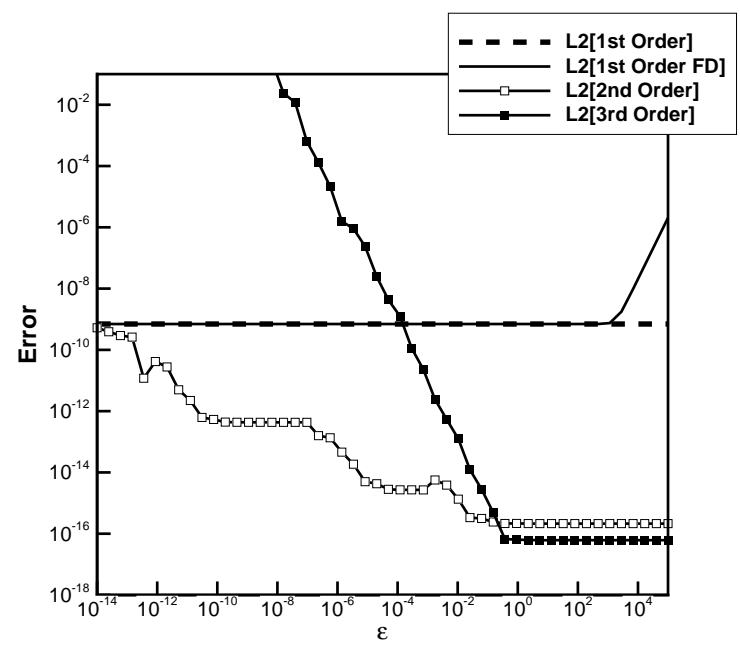

(a) $\eta=0.1$

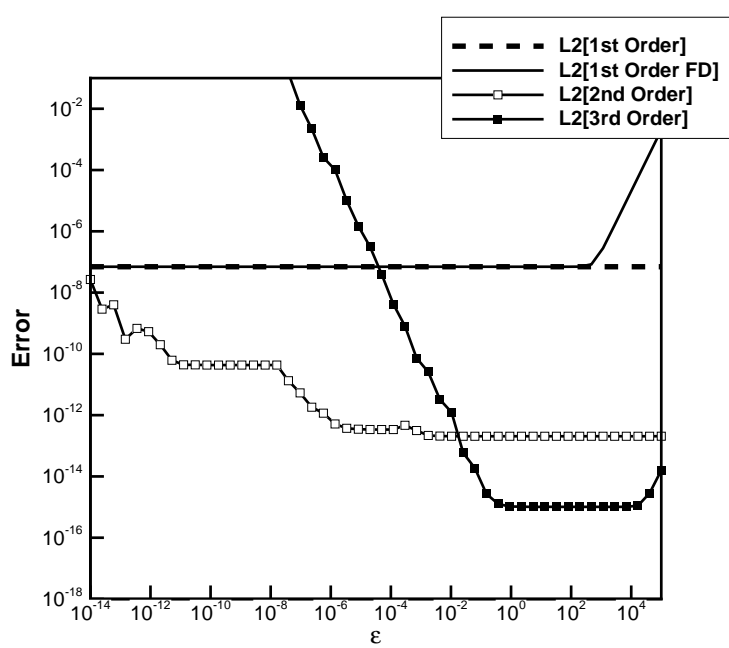

(b) $\eta=1.0$

Figure 11. L2 norm of the error in Taylor series truncation for several perturbation values, $\Delta w=\phi_{1} \cdot \eta$, around initial undeformed geometry; $\Phi_{1}$ is the first bending mode, and $\varepsilon$ is the step size used in the finite difference

\section{B. Forced-Response to a Harmonic Follower Force}

Forced-vibrations of the beam are driven by a harmonic follower force applied at the wing tip in the vertical direction ( $z$-axis in the beam reference system). The force is applied at the elastic axis avoiding any bending/torsion coupling. The force has the form

$$
F(t)=F_{0}+F_{A} \sin (\omega t)
$$

where $F_{0}$ is the static force and $F_{A}$ is the force amplitude with angular frequency $\omega$. These terms act here as free parameters so as the beam undergoes small/large deformations around small/large static deflections. In addition to the geometrically-exact nonlinear beam equations, the linearised equations are also available in analytic form. The approach used here and in the remaining sections is to perform first a linearised/nonlinear static solution, followed by the time-integration of the linearised/nonlinear dynamic equations. The integration in time of the large-order system uses the Newmark method. ${ }^{27}$ For ROM generation, two modes corresponding to the lowest bending modes were used.

The case for small deformations is examined first, $F_{A}=10 \mathrm{~N}$. Figure 12 shows the time history of the wing tip vertical displacement for two values of the applied static force. For $F_{0}=0 \mathrm{~N}$, the beam vibrates around its undeformed configuration, the response is linear and ROM predictions agree with the FOM responses. With a larger static deformation, Fig. 12(b), the linearized solution (labelled by "FOM") differs moderately from the nonlinear solution (labelled by "NFOM"). Two ROMs were generated. The curve "ROM" is the linear reduced model created at the static deformed position, which is assumed the reference geometry for model projection. The curve "NROM - 1st" was projected using the nonlinear beam equations and accounts 
for all applied forces that deform the beam from its undeformed geometry to the deformed position. Reduced models are in good agreement with the corresponding FOM responses.

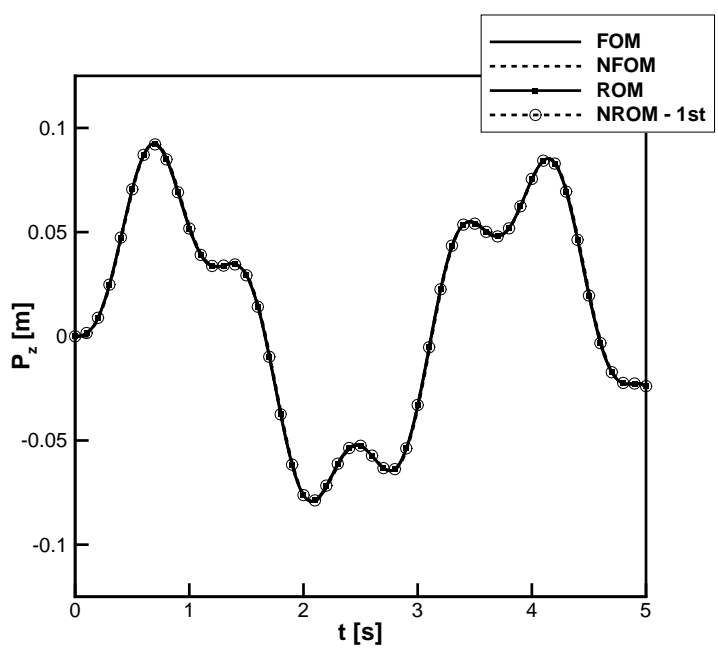

(a) $F_{0}=0 \mathrm{~N}$

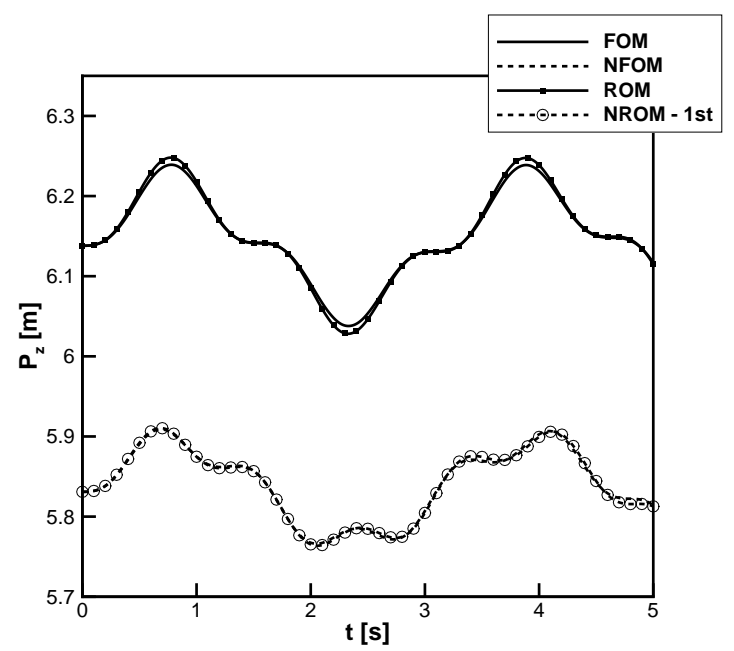

(b) $F_{0}=900 \mathrm{~N}$

Figure 12. Wing tip vertical displacement time response, $P_{z}(t)$, to a harmonic follower force $(\omega=2 \mathrm{rad} / \mathrm{s}$ and $F_{A}=10 \mathrm{~N}$ ); (b) beam deformations are large compared to the span of $16 \mathbf{m}$

Results for large deformations around a large static deflection are shown in Fig. 13, with the beam undergoing deflections of the order of $50 \%$ of its span. The beam deflection at every $0.5 \mathrm{~s}$ for the first 3 $\mathrm{s}$ is shown in Fig. 13(b) in scale 1:1 for the horizontal and vertical axes. The linearized solution differs substantially from the fully nonlinear solution. The curve labelled by "ROM" predicts well the response of the linearized system, referred to as "FOM". However, the interest here is on the performance of the nonlinear ROMs. Note that the curve "NROM - 1st" follows closely the nonlinear solution, and that the nonlinear ROM with quadratic terms is identical to the reference curve.

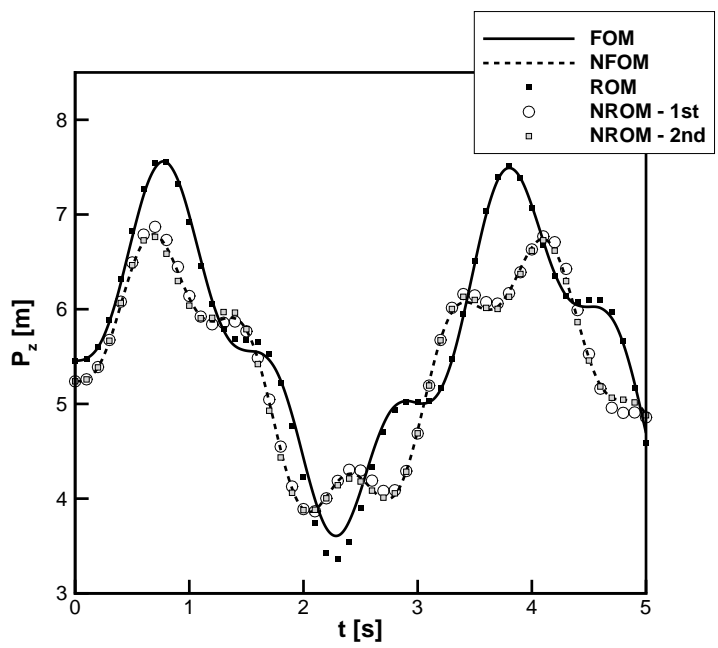

(a) $F_{0}=800 \mathrm{~N}$

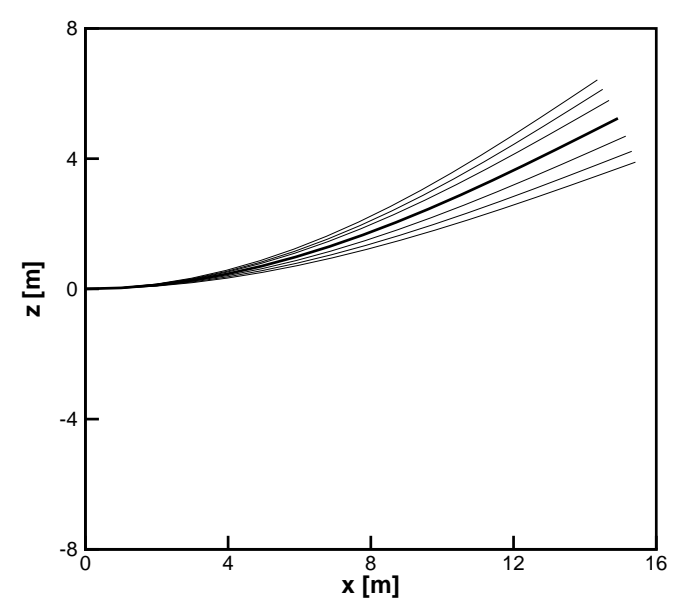

(b) Beam deformation

Figure 13. Wing tip vertical displacement time response, $P_{z}(t)$, to a harmonic follower force $(\omega=2 \mathrm{rad} / \mathrm{s}$ and $F_{A}=200 \mathrm{~N}$ ); (b) deformations obtained using nonlinear beam equations, the bold line is the static deformed position 


\section{Open-Loop Dynamic Aeroelastic Response to Gust}

The system derived from coupling the nonlinear beam code with two-dimensional potential flow is finally considered. To allow comparison with independent results, the freestream density $\rho_{\infty}=0.0899 \mathrm{~kg} / \mathrm{m}^{3}$ corresponding to an altitude of $20000 \mathrm{~m}$ is used. Tests were run to assess convergence of the results presented for increasing number of beam elements, and 16 elements were found adequate in all cases. Aerofoil sections are located at the position of structural nodes, and the coupled system comprises 20 degrees of freedom for each node, 12 arising from the structural model and 8 from the aerodynamic model.

The instability point of the coupled system can be identified rapidly using standard routines, as in the case of $\S \mathrm{V}$. The linear flutter speed and frequency are summarized in Table 3. For the reference data, ${ }^{26}$ an unsteady vortex-lattice method which accounts for three-dimensional effects at the wing tip was used. The favourable comparison shows that, for high-aspect ratio wings and high enough frequencies, two-dimensional aerodynamic models may be more cost-effective than 3-D methods.

\begin{tabular}{lcc}
\hline \hline & $U_{L}[\mathrm{~m} / \mathrm{s}]$ & $\omega[\mathrm{rad} / \mathrm{s}]$ \\
\hline Present & 102 & 69.7 \\
Ref. $^{26}$ & 104 & 72.4 \\
\hline \hline
\end{tabular}

Table 3. Linear flutter speed and frequency of the HALE wing $\left(h=20000 \mathbf{m}\right.$ and $\left.\rho_{\infty}=0.0899 \mathrm{~kg} / \mathbf{m}^{3}\right)$

The vertical displacement of the wing tip in response to gust perturbations of the form "1-cos" is shown in Fig. 14. Two cases are considered. The first is for $U_{\infty}=10 \mathrm{~m} / \mathrm{s}$ and the initial angle of attack before the gust encounter is $\alpha_{\infty}=10 \mathrm{deg}$. Because of the low dynamic pressure, loads acting on the wing are small and deflections remain linear. Following the penetration into the gust, the beam vibrates but the flow around it extracts energy and the response is damped. The basis for model generation includes several modes, of which two defining the lowest wing modeshapes. The second case is for a higher speed, $U_{\infty}=60 \mathrm{~m} / \mathrm{s}$. Differences between the linearized and nonlinear analyses increase for increasing dynamic pressure. For the linearized solution, the ROM prediction is virtually identical to the full-order solution. Nonlinear reduced models including up to quadratic terms follow closely the nonlinear solution.

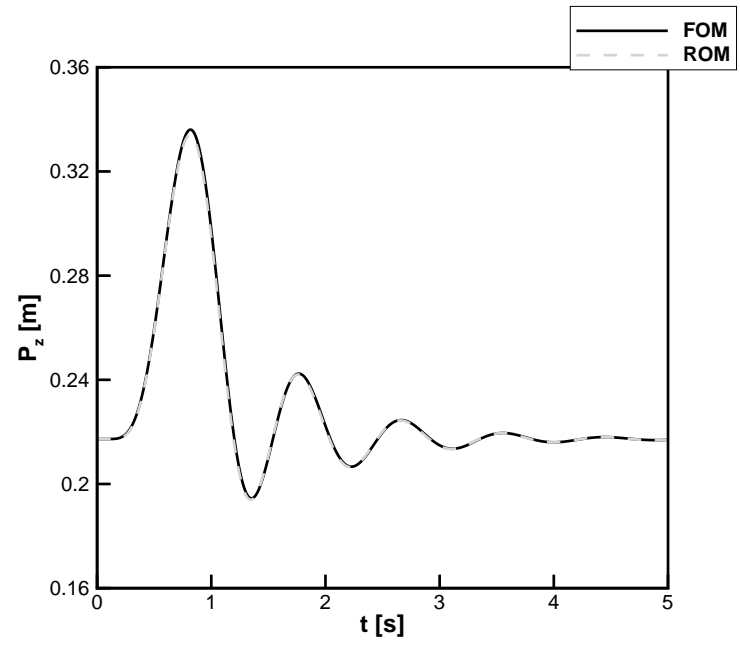

(a)

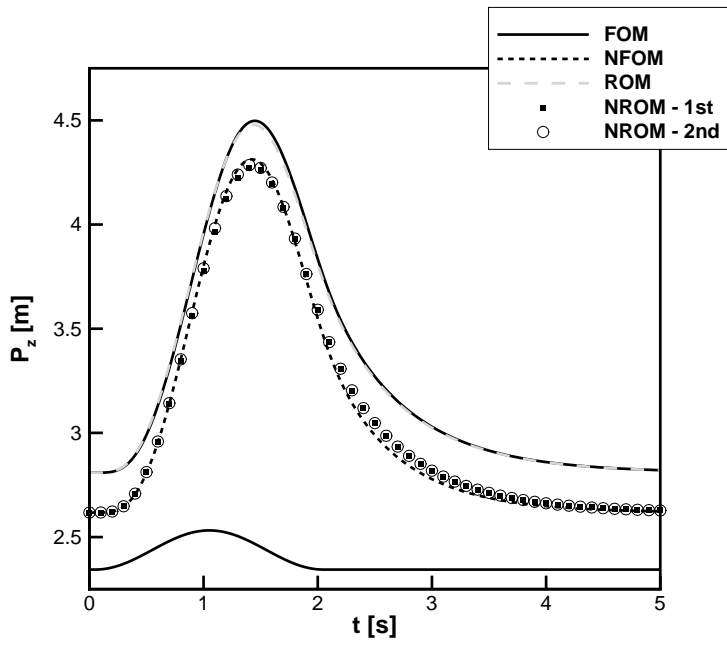

(b)

Figure 14. Wing tip vertical displacement time response, $P_{z}(t)$, to a "1-cos" gust shape of intensity $W_{0}=0.01$; (a) $\alpha_{\infty}=10 \mathrm{deg}, U_{\infty}=10 \mathrm{~m} / \mathrm{s}$, and $h_{g}=1 \mathrm{~m}$; (b) $\alpha_{\infty}=2.5 \mathrm{deg}, U_{\infty}=60 \mathrm{~m} / \mathrm{s}$, and $h_{g}=2 \mathrm{~m}$ 


\section{Conclusions}

A systematic approach to the model reduction of large dimension fluid-structure-flight models is outlined. The approach uses information on the eigenspectrum of the coupled system Jacobian matrix and projects the system through a series expansion onto a small basis of eigenvectors representative of the full-order dynamics. Results presented include:

- an aerofoil free to move in pitch and plunge with structural nonlinearities; the testcase is assumed as model problem to test the methods; cases with significant nonlinear structural effects were identified, and linear and nonlinear reduced models were generated to accurately predict the full-order dynamics; the design of a controller based on reduced models generated was shown to alleviate the gust loads;

- an aerofoil free to move in pitch and plunge with the flow modelled using the Euler equations; for large-order systems, the Schur complement was used to rapidly provide the information needed for model reduction, and various approximations to calculate the gust influence coefficient terms in the reduced model dynamics were examined;

- a flexible wing modelled using the geometrically-exact nonlinear beam equations; the coupled system was driven into small/large deformations around small/large static deflections, and reduced models were found to achieve a good agreement in all cases.

Future work will be addressed at exploiting the model reduction to control and alleviate the gust loads on the flexible wing and at testing the nonlinear model reduction when the flow is modelled using computational fluid dynamics equations. Then, the model reduction will be applied to nonlinear large order systems augmented with the rigid body dynamics.

\section{Acknowledgments}

This work was supported by the U.K. Engineering and Physical Sciences Research Council (EPSRC). The first author is also grateful to H. Hesse for his help with the nonlinear beam code and to N. Tantaroudas for several contributions.

For more details, the project webpage is http://www.cfd4aircraft.com/research_flexflight.html.

\section{References}

\footnotetext{
${ }^{1}$ Noll, T. E., Brown, J. M., Perez-Davis, M. E., Ishmael, S. D., Tiffany, G. C., and Gaier, M., "Investigation of the Helios Prototype Aircraft Mishap," NASA Tech. Rep., January, 2004.

${ }^{2}$ Palacios, R., Murua, J., and Cook, R., "Structural and Aerodynamic Models in Nonlinear Flight Dynamics of Very Flexible Aircraft," AIAA Journal, Vol. 48, No. 11, 2010, pp. 2648-2659, doi: 10.2514/1.52446.

${ }^{3}$ Silva, W., "Identification of Nonlinear Aeroelastic Systems based on the Volterra Theory: Progress and Opportunities," Nonlinear Dynamics, Vol. 39, No. 1-2, 2005, pp. 25-62, doi: 10.1007/s11071-005-1907-z.

${ }^{4}$ Da Ronch, A., Vallespin, D., Ghoreyshi, M., and Badcock, K. J., "Evaluation of Dynamic Derivatives Using Computational Fluid Dynamics," AIAA Journal, Vol. 50, No. 2, 2012, pp. 470-484, doi: 10.2514/1.J051304.

${ }^{5} \mathrm{Da}$ Ronch, A., McCracken, A., Badcock, K. J., Ghoreyshi, M., and Cummings, R. M., "Modeling of Unsteady Aerodynamic Loads," Atmospheric Flight Mechanics Conference, AIAA-2011-2376, Portland, Oregon, 2011.

${ }^{6}$ Lucia, D. J., Beran, P. S., and Silva, W. A., "Reduced-Order Modeling: New Approaches for Computational Physics," Progress in Aerospace Sciences, Vol. 40, No. 1-2, 2004, pp. 51-117, doi: 10.1016/j.paerosci.2003.12.001.

${ }^{7}$ Da Ronch, A., McCracken, A., Badcock, K. J., Widhalm, M., and Campobasso, M. S., "Linear Frequency Domain and Harmonic Balance Predictions of Dynamic Derivatives," Journal of Aircraft, Submitted for Publication 2011 [see also AIAA Paper 2010-4699].

${ }^{8}$ Woodgate, M. A. and Badcock, K. J., "Fast Prediction of Transonic Aeroelastic Stability and Limit Cycles," AIAA Journal, Vol. 45, No. 6, 2007, pp. 1370-1381, doi: 10.2514/1.25604.

${ }^{9}$ Badcock, K. J., Woodgate, M. A., Allan, M. R., and Beran, P. S., "Wing-Rock Limit Cycle Oscillation Prediction based on Computational Fluid Dynamics," Journal of Aircraft, Vol. 45, No. 3, 2008, pp. 954-961, doi: 10.2514/1.32812.

${ }^{10} \mathrm{Li}$, D., Guo, S., and Xiang, J., "Aeroelastic Dynamic Response and Control of an Airfoil Section with Control Surface Nonlinearities," Journal of Sound and Vibration, Vol. 329, 2010, pp. 4756-4771, doi: 10.1016/j.jsv.2010.06.006.

${ }^{11}$ Strganac, T. W., Ko, J., Thompson, D. E., and Kurdila, A. J., "Identification and Control of Limit Cycle Oscillations in Aeroelastic Systems," Journal of Guidance, Control, and Dynamics, Vol. 23, No. 6, 2000, pp. 1127-1133.

${ }^{12}$ Meirovitch, L., Dynamics and Control of Structures, Wiley, New York, 1989, pp. 93-98.

${ }^{13}$ Pettit, C. L. and Beran, P. S., "Effects of parametric uncertainty on airfoil limit cycle oscillation," Journal of Aircraft, Vol. 40, No. 5, 2003, pp. 1004-1006.
} 
${ }^{14}$ Theodorsen, T., "General Theory of Aerodynamic Instability and the Mechanism of Flutter," NACA Report Nr. 496, 1935.

${ }^{15}$ Jones, R. T., "The Unsteady Lift of a Wing of Finite Aspect Ratio," NACA Report Nr. 681, 1940.

${ }^{16}$ Leishman, J. G., "Unsteady Lift of a Flapped Airfoil by Indicial Concepts," Journal of Aircraft, Vol. 31, No. 2, 1994, pp. 288-297.

${ }^{17}$ Lee, B. H. K., Gong, L., and Wong, Y. S., "Analysis and Computation of Nonlinear Dynamic Response of a Two-Degreeof-Freedom System and Its Application in Aeroelasticity," Journal of Fluids and Structures, Vol. 11, No. 3, 1997, pp. 225-246, doi: $10.1006 /$ jfls.1996.0075.

${ }^{18}$ Liu, L., Wong, Y. S., and Lee, B. H. K., "Application of the Centre Manifold Theory in Non-Linear Aeroelasticity," Journal of Sound and Vibration, Vol. 234, No. 4, 2000, pp. 641-659, doi: 10.1006/jsvi.1999.2895.

${ }^{19}$ Hoblit, F. M., Gust Loads on Aircraft: Concepts and Applications, American Institute of Aeronautics and Astronautics, Reston, VA, USA, 1988.

${ }^{20}$ Kennett, D. J., Timme, S., Angulo, J., and Badcock, K. J., "An Implicit Meshless Method for Application in Computational Fluid Dynamics," International Journal for Numerical Methods in Fluids, 2012, doi: 10.1002/fld.3698.

${ }^{21}$ Badcock, K. J. and Woodgate, M. A., "Bifurcation Prediction of Large-Order Aeroelastic Models," AIAA Journal, Vol. 48, No. 6, 2010, pp. 1037-1046, doi: 10.2514/1.40961.

${ }^{22}$ Da Ronch, A., On the Calculation of Dynamic Derivatives Using Computational Fluid Dynamics, Ph.D. thesis, University of Liverpool, Liverpool, U.K., March 2012.

${ }^{23}$ Parameswaran, V. and Baeder, J. D., "Indicial Aerodynamics in Compressible Flow - Direct Computational Fluid Dynamic Calculations," Journal of Aircraft, Vol. 34, No. 1, 1997, pp. 131-133.

${ }^{24}$ Badcock, K. J., Woodgate, M., and Richards, B. E., "Hopf Bifurcation Calculations for a Symmetric Airfoil in Transonic Flow," AIAA Journal, Vol. 42, No. 5, 2004, pp. 883-892, doi: 10.2514/1.9584.

${ }^{25}$ Hodges, D. H., "A mixed variational formulation based on exact intrinsic equations for dynamics of moving beams," International Journal of Solids and Structures, Vol. 26, No. 11, 1990, pp. 1253-1273, doi: 10.1016/0020-7683(90)90060-9.

${ }^{26}$ Murua, J., Hesse, H., Palacios, R., and Graham, J. M. R., "Stability and Open-Loop Dynamics of Very Flexible Aircraft Including Free-Wake Effects," 52nd AIAA/ASME/ASCE/AHS/ASC Structures, Structural Dynamics and Materials Conference, AIAA-2011-1915, Denver, Colorado, 2011.

${ }^{27}$ Newmark, N. M., "A Method of Computation for Structural Dynamics," Journal of the Engineering Mechanics Division, Vol. 85, No. 3, 1959, pp. 67-94. 